\title{
A design principle for vascular beds: The effects of complex blood rheology
}

\author{
Tomás Alarcón ${ }^{\mathrm{a}, *}$, Helen M. Byrne ${ }^{\mathrm{b}}$, Philip K. Maini ${ }^{\mathrm{c}}$ \\ a Department of Computer Science, Bioinformatics Unit, University College, London, Gower Street, London WC1E 6BT, United Kingdom \\ ${ }^{\mathrm{b}}$ Centre for Mathematical Medicine, Division of Applied Mathematics, School of Mathematical Sciences, University of Nottingham, \\ Nottingham NG7 2RD, United Kingdom \\ ${ }^{\mathrm{c}}$ Centre for Mathematical Biology, Mathematical Institute, University of Oxford, 24-29 St. Giles', Oxford OX1 3LB, United Kingdom
}

Received 4 August 2004

Available online 24 March 2005

\begin{abstract}
We propose a design principle that extends Murray's original optimization principle for vascular architecture to account for complex blood rheology. Minimization of an energy dissipation function enables us to determine how rheology affects the morphology of simple branching networks. The behavior of various physical quantities associated with the networks, such as the wall shear stress and the flow velocity, is also determined. Our results are shown to be qualitatively and quantitatively compatible with independent experimental observations and simulations.
\end{abstract}

(C) 2005 Elsevier Inc. All rights reserved.

Keywords: Vascular remodeling; Network; Rheology; Poiseuille flow; Kirchoff's law

\section{Introduction}

The origins of and principles governing biological form have been a major scientific issue for many years. A particularly fruitful approach to the study of many morphogenetic processes is based on the hypothesis that they are regulated by physiological constraints that optimize function (LaBarbera, 1990). Several such principles have been proposed to explain the structure of the vascular system (Zamir, 1976), although the one which has received most attention from experimentalists and theoreticians is Murray's design principle. Murray used optimization arguments to develop laws governing branching in vascular trees (Murray, 1926). He proposed that the morphology of the vascular system should be determined by a process which minimizes the energy required to maintain the volume of blood and to drive the blood flow. While hydrodynamical resistance decreases as the diameter

\footnotetext{
* Corresponding author.

E-mail address: t.alarcon@cs.ucl.ac.uk (T. Alarcón).
}

of the vessel increases, more metabolic energy is required to sustain a larger volume of blood. The final geometry represents a balance between these competing effects, with lumen radius being proportional to the cubic root of the flow rate (Murray's law). Although the present article focuses on an optimization design principle, organization principles based on different assumptions can be formulated. For example, Gafiychuk and Lubashevsky (2001) showed Murray's law can be derived from a geometric argument, namely, that the vascular system has to be a space filling structure.

Murray's law has been tested extensively and appears to be valid in large arteries and arterioles (LaBarbera, 1990; Zamir, 1977). However, it does not provide a good description of the microcirculation (Frame and Sarelius, 1995; Pries et al., 1995). Zamir (1977) showed that Murray's design principle leads to a branching structure in which the wall shear stress (WSS) is constant. While the WSS is approximately constant in large arterial vessels, recent work by Pries et al. (1995) shows that it depends on the pressure inside the vessel: the WSS depends on the transmural pressure in a sigmoidal manner, saturating 
to a constant value for large pressures (i.e., large arteries and arterioles), as predicted by Murray's law.

In this paper, we develop a new design principle that can reproduce generic features of real vascular networks such as those reported in Pries et al. (1995). Taber (1998) has proposed a similar approach. His analysis is based upon the hypothesis that vasomotor control of blood flow requires a significant amount of the energy used to run the circulatory system. Accordingly, he modifies Murray's law to include energy costs associated with maintaining smooth-muscle contraction against the distending effects of the wall stress, which in turn depends on pressure and vessel geometry. The corresponding design principle yields a non-monotonic dependence of the WSS upon the pressure. There are two main differences between Taber's approach and that presented here. First, we neglect the energy costs associated with smooth-muscle maintenance, but we take into account other factors such as blood rheology. Second, whereas Taber (1998) deals with the behavior of a single vessel when the pressure is modified, we build a network according to our design principle and analyze the behavior of the WSS across the network. Hence, our approach is more closely related to the experimental procedure of Pries et al. (1995). Our design principle produces a new (local) law relating the lumen radius at branch points that provide a more realistic description of vascular trees than that obtained from Murray's law; this issue is not addressed in Taber (1998).

The paper is organized as follows. In Materials and methods, we present our mathematical model and all the elements we use to analyze it and to interpret our results. Results is devoted to a description of our results. In the Conclusions section, our conclusions are presented.

\section{Materials and methods}

In this section, we use a design principle as our basic mathematical model to explain a number of properties of the vascular system such as those observed by Pries et al. (1995). We begin with a brief description of Murray's law. We then show how it may be modified in order to obtain more realistic results. We claim that this can be accomplished by including in the design principle key characteristics of blood flow and vessel behavior, namely, complex blood rheology.

\section{Mathematical model: design principle}

Murray's law

As stated above, Murray's law establishes that the vascular tree is organized so that a balance exists between the metabolic energy of a given volume of blood and the energy required for blood flow. Specifically, it is based on a minimization principle for the dissipated power $W$. The flow is assumed to be Poiseuille, the vessels are considered rigid tubes and the pressure gradient is assumed to be constant. The blood is viewed as a Newtonian fluid of constant viscosity. These assumptions lead to the following formulation of the design principle for a single vessel of length $L$ and radius $R$ :

$\frac{\partial W}{\partial R}=0$

$W=W_{\mathrm{H}}+W_{\mathrm{M}}$,

$W_{\mathrm{H}}=\frac{8 \dot{Q}^{2} \mu_{0} L}{\pi R^{4}}$

$W_{\mathrm{M}}=\alpha_{\mathrm{b}} \pi R^{2} L$.

In Eq. (1), $W_{\mathrm{H}}$ is the power dissipated by the flow (Fung, 1993), $W_{\mathrm{M}}$ is the metabolic energy consumption rate of the blood, $\dot{Q}$ is the flow rate, $\mu_{0}$ is the blood viscosity, and $\alpha_{\mathrm{b}}$ is the metabolic rate per unit volume of blood. Using Eq. (1), we deduce that the total dissipated power $W$ is minimized if

$\dot{Q}=\frac{\pi R^{3}}{4} \sqrt{\frac{\alpha_{\mathrm{b}}}{\mu_{0}}}$

Eq. (2) shows how the vessel radius $R$ and the flow rate $\dot{Q}$ are related when the optimization principle is satisfied. Additionally, at a bifurcation in the vascular tree, such as the one depicted in Fig. 1, conservation of mass implies

$\dot{Q}_{\mathrm{p}}=\dot{Q}_{1}+\dot{Q}_{2}$

where $\dot{Q}_{p}$ is the flow rate through the parent vessel. Taken together, Eqs. (2) and (3) imply that, at a bifurcation, the

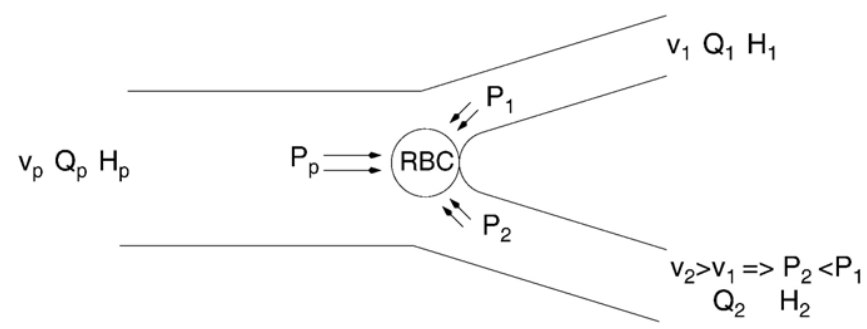

Fig. 1. Schematic diagram of a bifurcation. $H_{\mathrm{p}}, P_{\mathrm{p}}, \dot{Q}_{\mathrm{p}}$, and $v_{0}$ are the hematocrit, pressure, flow rate, and flow velocity in the parent vessel, and $H_{i}, P_{i}, \dot{Q}_{i}$, and $v_{i}, i=1,2$, are the hematocrit, pressure, flow rate, and flow velocity in each one of the daughter branches. RBC stands for red blood cell. The arrows indicate the direction in which the (local) pressure acts on the RBC. 
radius of the parent vessel, $R_{\mathrm{p}}$, and the radii of the daughter vessels, $R_{1}$ and $R_{2}$, satisfy

$R_{\mathrm{p}}^{3}=R_{1}^{3}+R_{2}^{3}$.

This is Murray's basic result concerning the architecture of the vascular tree. Using the above result, it is easy to determine the WSS within a given vessel. For Poiseuille flow, the WSS is given by

$\tau_{w}=\frac{4 \mu_{0} \dot{Q}}{\pi R^{3}}=\sqrt{\alpha_{\mathrm{b}} \mu_{0}}$,

using Eq. (2).Thus, we conclude that the WSS is constant throughout vascular networks constructed according to Murray's principle (Zamir, 1977).

\section{Effects of blood rheology}

Murray's law neglects certain factors, such as rheology, which may play an important role in the organization of the vascular tree (Fung, 1993; Pries et al., 1994). Blood is far from being a simple fluid with constant viscosity: it is a very complex suspension of cells and molecules of a wide range of sizes. Consequently, treating blood as a Newtonian fluid is a very crude approximation. Fortunately, there are experimental results which provide justification for neglecting many of these features, enabling us to focus on the key role played by red blood cells in blood rheology. As shown in Fig. 2, the relative (non-dimensional) blood viscosity, $\mu_{\text {rel }}(R, H)$, depends on $R$ and also on the hematocrit $H$, which is defined as the ratio between the total blood volume and the volume occupied by red blood cells. The relative viscosity exhibits a non-monotonic dependence on $R$ which can be sub-divided into three different regions. If $R$ is much greater than the typical size of a red blood cell ${ }^{1}$, then the viscosity is independent of the vessel radius. As the radius of the vessel decreases, the viscosity also decreases (the Fahraeus-Lindqvist effect) until the viscosity attains a minimum value $R \sim 25 \mu \mathrm{m}$. For smaller values of $R$, the viscosity increases as $R$ decreases (Pries et al., 1994). The dependence on the hematocrit is easier to understand: the higher is the hematocrit, the thicker the blood becomes, and therefore its viscosity increases.

In this paper, we take $\mu(R, H)=\mu_{0} \mu_{\text {rel }}(R, H)$, where $\mu_{0}$ is the viscosity of plasma and $\mu_{\text {rel }}$ is the (non-dimensional) relative viscosity. In particular, we follow Pries et al. (1994), assuming (see Fig. 2)

$\mu_{\text {rel }}=\left[1+\left(\mu_{0.45}^{*}-1\right) \frac{(1-H)^{C}-1}{(1-0.45)^{C}-1}\left(\frac{2 R}{2 R-1.1}\right)^{2}\right]\left(\frac{2 R}{2 R-1.1}\right)^{2}$,

$\mu_{0.45}^{*}=6 \mathrm{e}^{-0.17 R}+3.2-2.44 \mathrm{e}^{-0.06(2 R)^{0.645}}$,

$C=\left(0.8+\mathrm{e}^{-0.15 R}\right)\left(-1+\frac{1}{1+10^{-11}(2 R)^{12}}\right)+\frac{1}{1+10^{-11}(2 R)^{12}}$.

The radius $R$ is given in $\mu \mathrm{m}$.

\footnotetext{
${ }^{1}$ Average red blood cell diameter in humans is $7-8 \mu \mathrm{m}$.
}

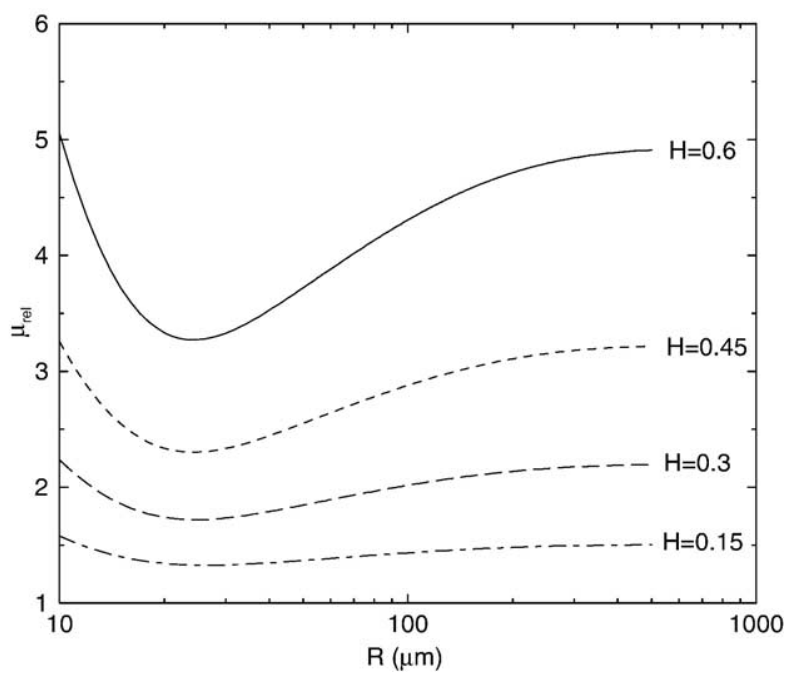

Fig. 2. Graph showing how the relative viscosity $\mu_{\text {rel }}$ varies with the vessel radius $R$ for fixed values of the hematocrit $H$ (see Eq. (6)).

\section{The design principle}

We now formulate our design principle as follows:

$\frac{\partial W}{\partial R}=0$,

$W=W_{\mathrm{H}}+W_{\mathrm{M}}$,

$W_{\mathrm{H}}=\frac{8 \dot{Q}^{2} \mu_{0} \mu_{\mathrm{rel}}(R, H) L}{\pi R^{4}}$,

$W_{\mathrm{M}}=\alpha_{\mathrm{b}} \pi R^{2} L$

In Eq. (7), $\mu_{0}$ is the viscosity of plasma and $\mu_{\text {rel }}(R, H)$ is given by Eq. (6). Substituting from Eq. (6) into Eq. (7), we deduce that the optimization principle predicts that $\mathrm{Q}^{\circ}$ and $R$ are related as follows:

$\dot{Q}=R^{3} \frac{\pi}{2 \mu_{0}^{1 / 2}} \sqrt{\frac{\alpha_{\mathrm{b}}}{4 \mu_{\mathrm{rel}}-R \partial \mu_{\mathrm{rel}} / \partial R}}$.

As a first approximation, we assume that $H$ is fixed in Eq. (6) and consider the corresponding results with a radiusdependent viscosity only. In particular, we have set $H=$ 0.45 . This particular value has been chosen because in Pries et al. (1994), $H=0.45$ is set as a "reference value "in the fitting procedure to obtain Eq. (6). In this case, when we solve Eq. (8) numerically and plot the results on a $\log -\log$ scale (see Fig. 3), we observe three different behaviors. For large flow rates, $\dot{Q} \sim R^{3.00}$, for intermediate values $\dot{Q} \sim$ $R^{2.87}$, whereas for small flow rates $\dot{Q} \sim R^{3.66}$. The regions in which the data may be described by power laws are separated by cross-over regions where $R \sim R_{\text {crl }}(\dot{Q} \sim$ $\left.\dot{Q}_{c r 1}\right)$ and $R \sim R_{\mathrm{cr} 2}\left(Q \sim \dot{Q}_{c r 2}\right)$. In these transition regions, the power law fails to describe the data. 
(a)

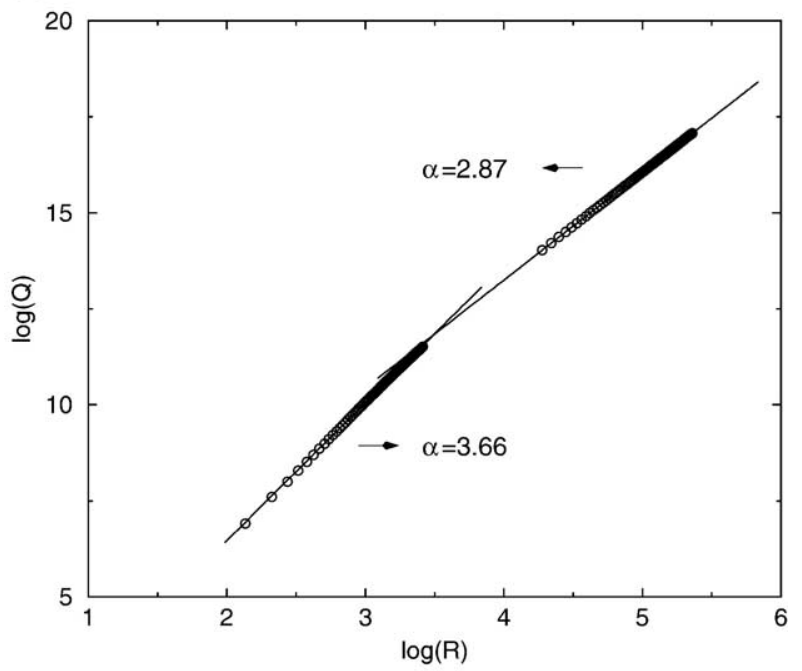

(b)

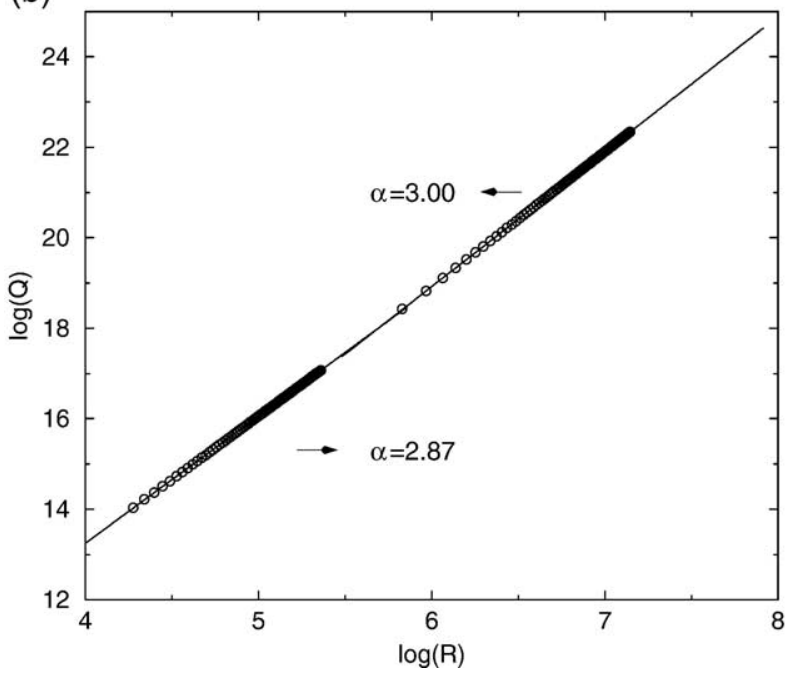

Fig. 3. Dependence of the flow rate $(\dot{Q})$ on the radius $(R)$ in an optimal network (see Eq. (8)). We have assumed the hematocrit is constant, $H=0.45$.

Using Eq. (8) and mass conservation, we can derive a relationship between vessel radii at bifurcation points, as $\dot{Q}=$ $C R^{\alpha}$ in each one of the regions mentioned in the previous paragraph. Recall that for Murray's law we have

$$
\begin{aligned}
C\left(R_{p}, H_{p}\right) R_{p}^{\alpha\left(R_{p}, H_{p}\right)}= & C\left(R_{1}, H_{1}\right) R_{1}^{\alpha\left(R_{1}, H_{1}\right)} \\
& +C\left(R_{2}, H_{2}\right) R_{2}^{\alpha\left(R_{2}, H_{2}\right)},
\end{aligned}
$$

with $\alpha\left(R_{i}, H_{i}\right)=3$ and $C\left(R_{i}, H_{i}\right)=C\left(R_{j}, H_{j}\right)$ with $i, j=p$, 1, 2. A similar relationship can be obtained in the present case. Indeed, as $R \sim \dot{Q}^{1 / \alpha}$, Eq. (9) also applies in the present case but the exponents $\alpha_{i}$ and the coefficients $C_{i}$ depend on the radius of the vessel in the following manner:

$\alpha_{i}=\left\{\begin{array}{c}3.00 \text { if } R_{1}>R_{c r 1}, \\ 2.87 \text { if } R_{c r 2} \leq R_{i} \leq R_{c r 1}, \\ 3.66 \text { if } R_{i}<R_{c r 2},\end{array}\right.$ and

$C_{i}=\left\{\begin{array}{c}2.65 \text { if } R_{1}>R_{c r 1}, \\ 7.53 \text { if } R_{c r 2} \leq R_{i} \leq R_{c r 1}, \\ 0.41 \text { if } R_{i}<R_{c r 2},\end{array}\right.$

where $R_{c r 1} \simeq 270 \mu \mathrm{m}$ and $R_{c r 2} \simeq 45 \mu \mathrm{m}$.

The values of the coefficients $\alpha_{i}$ and $C_{i}$ were calculated for the particular values of the experimentally determined parameters stated in Table 1 and Eq. (6). A discussion of how sensitive our results are to changes in parameters is given in link: Qualitative analysis from model viscosities.

Taken together, Eq. (9) shows that we recover Murray's result for large vessels with a large flow rate. However, the behavior is different in smaller vessels.

\section{Qualitative analysis from model viscosities}

The previous section was based on experimental data for the viscosity and, in particular, an expression relating the viscosity to the vessel radius and the hematocrit obtained by Pries et al. (1995). The complexity of Eq. (6) renders analytical progress extremely difficult. Therefore, in this section, we incorporate the generic features of blood viscosity into simpler expressions which we use to gain a deeper understanding of our results and to identify conditions under which they are relevant. We also investigate those properties of viscosity which, when incorporated into the design principle, give rise to experimentally observed behavior and, in particular, the sigmoidal dependence of the WSS on the transmural pressure.

One of the simplest model (relative) viscosities one can imagine is the piece-wise approximation (see Fig. 4),

$\mu_{\mathrm{rel}}(R, H)=\left\{\begin{array}{l}\mu_{\mathrm{sat}}(H)\left(\frac{R_{\min }}{R_{\mathrm{sat}}}\right)^{\gamma(H)}\left(\frac{R}{R_{\mathrm{min}}}\right)^{-\beta(H)} \text { if } R \leq R_{\mathrm{min}}, \\ \mu_{\text {sat }}(H)\left(\frac{R}{R_{\mathrm{sat}}}\right)^{\gamma(H)} \text { if } R_{\mathrm{min}} \leq R \leq R_{\mathrm{sat}}, \\ \mu_{\mathrm{sat}}(H) \text { if } R \geq R_{\mathrm{min}}\end{array}\right.$

The physical meanings of the different parameters appearing in Eq. (12) are shown in Fig. 4. Additionally, $\mu_{\text {sat }}, \beta$, and $\gamma$ areas assumed to be increasing functions of the

Table 1

\begin{tabular}{|c|c|c|c|}
\hline Parameter & Value & Units & Source \\
\hline$\mu_{0}$ & 1.2 & $\mathrm{cP}$ & Fung (1990) \\
\hline$\alpha_{b}$ & 778 & $\operatorname{erg~s}^{-1} \mathrm{ml}^{-1}$ & Taber (1998) \\
\hline$R_{\min }$ & 25 & $\mu \mathrm{m}$ & Estimated from Eq. (6) \\
\hline$R_{\text {sat }}$ & 100 & $\mu \mathrm{m}$ & Estimated from Eq. (6) \\
\hline $\begin{array}{l}l_{\text {sat }} \\
\gamma(H)\end{array}$ & $1.38 H$ & & Estimated from Eq. $(6)^{\mathrm{a}}$ \\
\hline$\beta(H)$ & $0.04 H+13.7 H^{2}$ & & Estimated from Eq. $(6)^{\mathrm{a}}$ \\
\hline$\mu_{\text {sat }}(H)$ & $1.00+2.79 H+3.67 H^{2}$ & & Estimated from Eq. (6) \\
\hline
\end{tabular}

Values of the parameters we have used in our simulations 


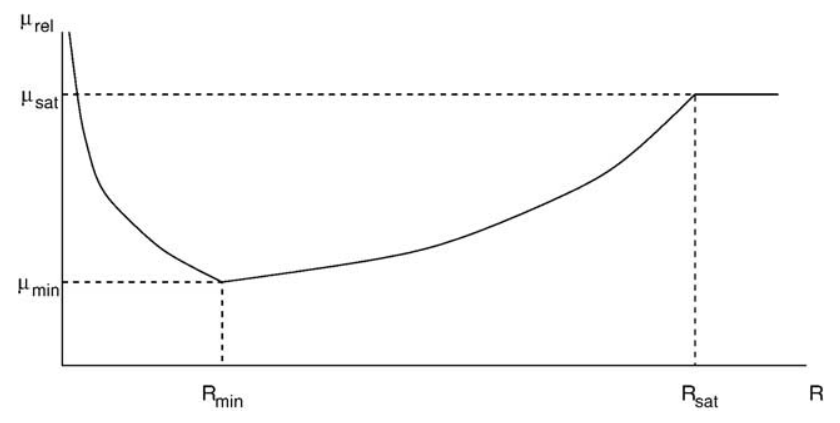

Fig. 4. Schematic representation of our simplified model with a piece-wise continuous viscosity.

hematocrit (see Fig. 2) while $R_{\min }$ and $R_{\text {sat }}$ are assumed to be constants, although a closer look at Fig. 2 reveals that this assumption is more accurate for $R_{\min }$ than for $R_{\mathrm{sat}}$. However, this notoriously simplifies the algebraic manipulation. This example will allow us to compare some qualitatively different situations and hence to predict the mechanisms that give rise to the observed dependence of the WSS on the transmural pressure.

We substitute Eq. (12) into Eq. (8) to obtain:

$\dot{Q}=\left\{\begin{array}{l}\frac{\pi}{2} \sqrt{\frac{\alpha_{\mathrm{b}}}{(4+\beta(H)) \mu_{0} \mu_{\mathrm{sat}}(H)}\left(\frac{R_{\mathrm{sat}}}{R_{\min }}\right)^{\gamma(H)}} \frac{R^{3}+\beta(H) / 2}{R_{\min }^{\beta(H) / 2}} \text { if } R \leq R_{\min }, \\ \frac{\pi}{2} \sqrt{\frac{\alpha_{\mathrm{b}}}{(4-\gamma(H)) \mu_{0} \mu_{\mathrm{sat}}(H)}} \frac{R^{3}-\gamma(H) / 2}{R_{\min }^{-\gamma(H) / 2}} \text { if } R_{\min } \leq R \leq R_{\mathrm{sat}}, \\ \frac{\pi}{2} \sqrt{\frac{\alpha_{\mathrm{b}}}{4 \mu_{0} \mu_{\mathrm{sat}}}} R^{3} \text { if } R \geq R_{\min },\end{array}\right.$

so that

$\alpha=\left\{\begin{array}{l}3+\beta(H) / 2 \text { if } R \leq R_{\min }, \\ 3-\gamma(H) / 2 \text { if } R_{\min } \leq R \leq R_{\text {sat }}, \\ 3 \text { if } R \geq R_{\text {sat }}\end{array}\right.$

and

$C=\left\{\begin{array}{l}\frac{\pi}{2} \sqrt{\frac{\alpha_{\mathrm{b}}}{(4+\beta(H)) \mu_{0} \mu_{\mathrm{sat}}(H)}\left(\frac{R_{\mathrm{sat}}}{R_{\min }}\right)^{\gamma(H)}} \frac{1}{R_{\min }^{\beta(H) / 2}} \text { if } R \leq R_{\mathrm{min}}, \\ \frac{\pi}{2} \sqrt{\frac{\alpha_{b}}{(4-\gamma(H)) \mu_{0} \mu_{\mathrm{sat}}(H)}} \frac{1}{R_{\min }^{-\gamma(H) / 2}} \text { if } R_{\min } \leq R \leq R_{\mathrm{sat}}, \\ \frac{\pi}{2} \sqrt{\frac{\alpha_{\mathrm{b}}}{4 \mu_{0} \mu_{\mathrm{sat}}}} \text { if } R \geq R_{\min } .\end{array}\right.$

Notice that from Eqs. (13) and (15) the condition $\gamma<4$ has to be satisfied.

\section{Simulations}

In order to determine whether our design principle accounts for the experimental results of Pries et al. (1995), we carried out numerical simulations for simple branching networks such as the one shown in Fig. 5, its morphology being determined by the optimization principle discussed in the preceding section.

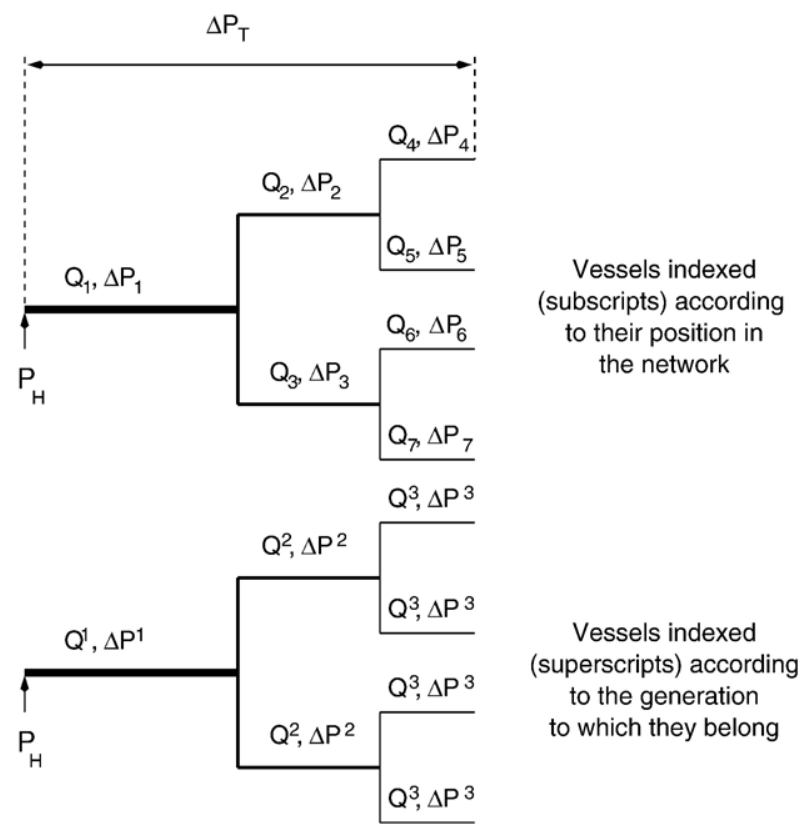

Fig. 5. Schematic representation of the branching structure used in our simulations. See text for details.

The system under investigation is a branching network of vessels. We assume that at each generation all vessels are identical and that their number increases by a factor 2 , i.e., the number of vessels, $N^{k+1}$, in the $(k+1)$ th generation is $N^{k+1}=$ $2 N^{k}$, where $N^{k}$ is the number of vessels in generation $k$. Their lengths are set according to $L^{k+1}=\rho L^{k}$ with $\rho \leq 1$ (West et al., 1997) and their radii are given by Eq. $(9)^{2}$.

As we are assuming that the flow in each vessel is incompressible, the flow pattern in this network satisfies Kirchoff's laws, i.e., flow conservation at each bifurcation point,

$\dot{Q}_{i}=\dot{Q}_{j}+\dot{Q}_{k}$,

where $j$ and $k$ denote vessels branching from vessel $i$, and conservation of energy

$\sum_{j} \Delta P_{j}=\Delta P_{\mathrm{T}}$

In Eq. (17), $\Delta P_{\mathrm{T}}$ is the pressure drop across the whole network, $\Delta P_{j}$ is the pressure drop across vessel $j$, and the sum is over the vessels in a continuous path connecting the ends of the network (see Fig. 5). Additionally, we assume Poiseuille flow in each vessel, so that

$\Delta P_{j}=Z_{j} \dot{Q}_{j}$,

where $Z_{j}=8 \mu_{0 \mu_{\mathrm{rel}}}\left(R_{j}, H_{J}\right) L_{j} / \pi R_{j}^{4}$.

Eqs. (16)-(18) fully determine the flow rate in all the vessels of the network, provided we know their radii and

\footnotetext{
${ }^{2}$ Throughout the rest of the paper, we adopt the following notation: superscripts denote the generation or level in the branching network, whereas subscripts denote a particular vessel in the tree (see Fig. 5 for the numbering scheme).
} 
lengths. In Appendix A, we explain how the geometry of the vessels can be determined according to the restrictions imposed by our optimization principle. In broad terms, we use Eqs. (16) and (17) to obtain the flow rate in each vessel and Eq. (18) to determine the pressure drop across each vessel of the network. This enables us to calculate the pressure $^{3}$ in any vessel belonging to generation $k$ as

$P^{k}=P_{\mathrm{H}}-\sum_{i=1}^{k-1} \Delta P^{i}-\frac{\Delta P^{k}}{2}$,

where $P_{\mathrm{H}}$ is the pressure at the inlet point of the network. Further, given the radii and flow rates in each vessel, we can use Eq. (5) to calculate the WSS. Eqs. (18) and (19) provide a relation between pressure and radius. Since there is a oneto-one correspondence between a vessel's generation and its radius, we can use this relation to plot the WSS as a function of the pressure.

Another factor that needs to be accounted for is hematocrit distribution at bifurcations. According to Fung (1993), the simplest way to proceed is to assume that the distribution of hematocrit depends on the flow velocity of the daughter vessels. Roughly speaking, a larger proportion of the hematocrit from the parent vessel, $H_{\mathrm{p}}$, is transported along the faster branch (see Fig. 1):

$H_{\mathrm{p}}=H_{1}+H_{2}$

$\frac{H_{1}}{H_{2}}=\frac{v_{1}}{v_{2}}$

where $v_{i}=\dot{Q}_{i} / \pi R_{i}^{2}(i=1,2)$. Eq. (20) is a simplification of the expression given by Fung (1984) in order to simplify the algebraic manipulation, althxough in the case of networks in which all the bifurcations are symmetrical, which is analyzed in detail in Results, this may not yield totally realistic results (for example, hematocrit values are very small after a large number of bifurcations).

To verify our procedure, we solved Kirchoff's laws for a network constructed according to Murray's law, i.e., $\alpha_{i}=3$, $i=1,2,3$. We found that $\dot{Q} \sim R_{3}$ and $\tau_{w}$ was constant, as expected.

\section{Results}

\section{Symmetrically branching network with uniform hematocrit}

In Appendix A, we show that a branching network, in which all vessels in the same generation are identical, satisfies the restrictions imposed by our design principle. Hereafter such a network is referred to as a symmetrically branching

\footnotetext{
${ }^{3}$ We compute the pressure at the mid-point of the vessel. As we are assuming that the vessels are rigid, the pressure at a point $x$ along the axis of the vessel is simply given by $P(x)=P_{\mathrm{H}}-(\Delta P / L) x$. We will assume that all the vessels in a given generation are identical and therefore the corresponding pressures are also identical.
}

network. Our aim in this section is to test our model in the simple situation when variation in hematocrit is not taken into account, so that blood viscosity depends on the radius in the non-monotonical way shown in Fig. 4 but is independent of the hematocrit. For the simulations discussed in this section, we take $\mu(R) \equiv \mu_{0 \mu_{\text {rel }}}(R, H=0.45)$.

Initially, we present results for a symmetrically branching network. As shown in Appendix A, this network is not unique. Due to the non-monotonic dependence of the viscosity upon the radius, we can construct asymmetrically branching networks. Hence, although the results we discuss in this section are obtained for a simple case, our model is also able to produce heterogeneous structures. Such behavior is consistent with experimental results that indicate that no two vascular trees are identical. This point will be discussed further in The role of hematocrit: asymmetric bifurcations.

We perform simulations on optimal symmetric networks, whose structure is determined by the branching exponents of Eq. (13). For simplicity, we use the simplified model relative viscosity (Eq. (12)) rather than Eq. (6). The branching exponents of Eq. (13) are obtained by fixing $H=0.45$ in Eq. (6). If we fix $H=0.45$ in Eq. (13), by comparison to Eq. (6), we can obtain the corresponding values of $\beta(H=0.45)=$ 2.81 and $\gamma(H=0.45)=0.62$.

The rest of the parameters needed to determine the relative viscosity (Eq. (12)) are obtained from Eq. (6) with $H=0.45$. We will take $R_{\text {min }}=25 \mu \mathrm{m}, R_{\text {sat }}=100 \mu \mathrm{m}$, and $\mu_{\text {sat }}(H=0.45)=3.2$. The network is generated according to Eq. (A.16). We have taken $R^{1}=350 \mu \mathrm{m}, L^{1}=0.7 \mathrm{~cm}, P_{\mathrm{H}}=$ $100 \mathrm{~mm} \mathrm{Hg}$, and $\Delta P_{\mathrm{T}}=80 \mathrm{~mm} \mathrm{Hg}$.

We have carried out simulations for $\rho=1$, so that all the vessels in the network have the same length (results shown in Fig. 6). All the vessels within a generation have the same radius. The results of these numerical computations are illustrated in Fig. 6. We compare these results to the results reported by Pries et al. (1995) (Figs. 1, 3, and 5).

Our predictions of the wall shear stress as a function of the pressure exhibit a behavior resembling the experimental observations. If we compare our simulation results for $\rho=1$ (Fig. 6a) and the data shown in Pries et al. (1995), Fig. 3 for arterioles (we are considering a vascular tree, not a full vascular network with a venous side), the 5-fold drop in the wall shear stress we observe is consistent with the average drop observed experimentally (see Pries et al., 1995; Fig. 3).

Simulation results for the radius as a function of pressure (Fig. 6c) are in good agreement with the observations (see Pries et al., 1995; Fig. 5). The relationships we obtain between flow velocity and vessel radius are also in qualitative agreement (compare Fig. 6b with Pries et al., 1995, Fig. 1), although concavity is lacking. However, quantitative agreement is poor: we are predicting flow velocities two orders of magnitude higher than those observed experimentally.

The results obtained for $v(R)$ for small vessels might result a bit surprising. However, the origins of this non-monotonic dependence of $v$ on $R$ can be easily understood in terms of the 


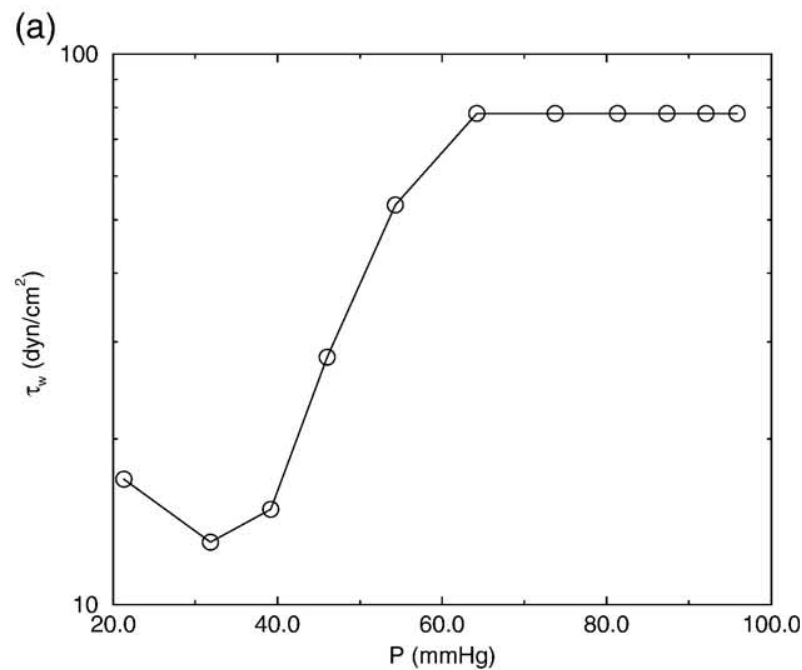

(b)

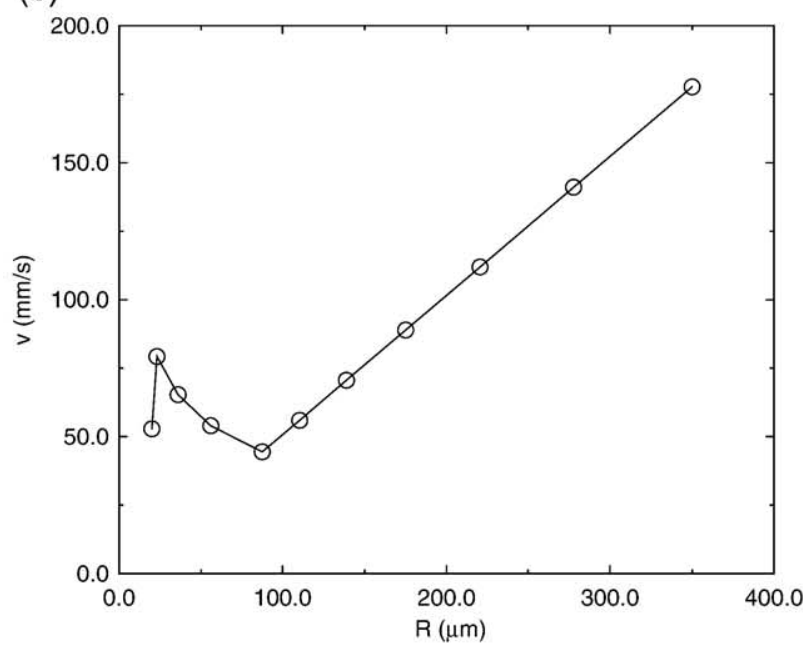

(c)

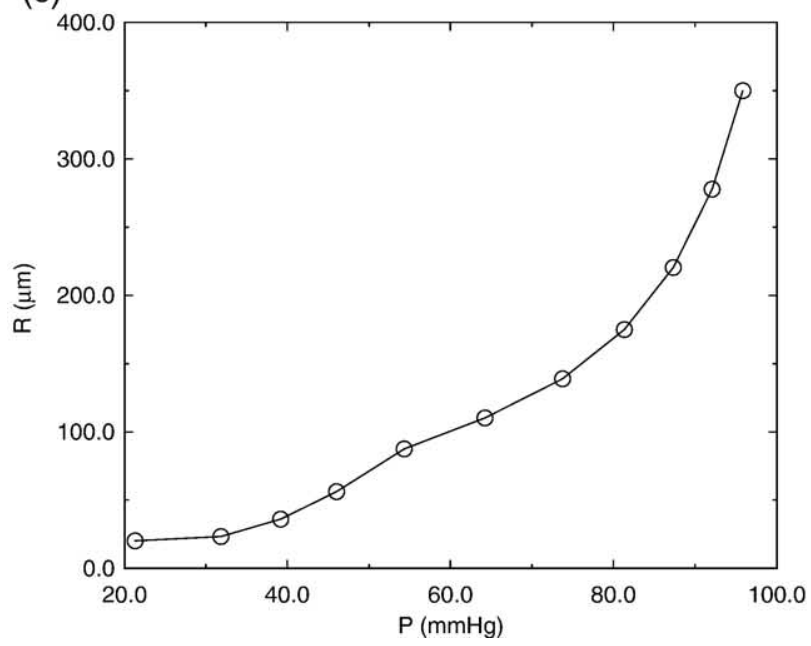

Fig. 6. Simulation results for a symmetrically branching network with $\rho=$ 1, i.e., all the vessels have the same length. Constant hematocrit is assumed. Panels $\mathrm{a}$ and $\mathrm{b}$ show the wall shear stress as a function of the pressure and the flow velocity as a function of the radius, respectively. Panel c shows the radius as a function of the pressure. The viscosity is given by Eq. (12) with $H=0.45$. See text for parameter values. corresponding form of $\mu(R)$. For $R<R_{\min }, \mu(R)$ decreases as a function of $R$, whereas for $R_{\min }<R<R_{\text {sat }}, \mu(R)$ increases. Accordingly, as $R$ increases, so does $v$ as long as $R<R_{\min }$. When $R_{\min }<R<R_{\text {sat }} \mu(R)$, as the viscosity increases, $v$ decreases. For $R>R_{\text {sat }}$, the viscosity is kept constant but the resistance $Z \sim \mu_{\text {sat }} / R^{4}$ is a decreasing function of $R$ and therefore $v$ increases. Note that the boundaries between the regions in which $v(R)$ shows these different behaviors correspond to $R_{\min }$ and $R_{\text {sat }}$.

It might be surprising that we obtain 5 -fold to 8 -fold reductions in the WSS for a small change in the value of the relative viscosity ${ }^{4}$. However, for example in the case $\rho=1$, by comparing $\tau\left(R_{\text {sat }}\right)$ and $\tau\left(R_{\min }\right)$, we obtain (see Eqs.), (12) and (13):

$\frac{\tau\left(R_{\min }\right)}{\tau\left(R_{\mathrm{sat}}\right)}=(4-\gamma(H))^{-1 / 2}\left(\frac{R_{\mathrm{min}}}{R_{\mathrm{sat}}}\right)^{\gamma(H)} \simeq 0.23$,

where we have used the parameter values given in Table 1 . This result is consistent with our simulations.

\section{The role of hematocrit: asymmetric bifurcations}

So far, we have presented results concerning symmetrically branching networks with uniform hematocrit, assuming only a non-monotonical dependence of the viscosity on the radius of the vessel (Fig. 4). Although our results appear to capture some of the experimentally observed trends, our approach so far has one serious weakness: it implies a very tight control system for the developing vasculature and leaves no room for noise or variability in the structure of the vascular network.

As we show in Appendix A, by formulating our design principle in the form of a relationship between radius and blood flow, the final structure of the network is imposed by conservation laws. In the uniform hematocrit case, the only possible source of heterogeneity is a rather narrow window where asymmetric bifurcations are possible (see Appendix A for details).

However, the inclusion of variable hematocrit naturally introduces a source of heterogeneity into the possible structures our branching network can exhibit. As we show in Appendix A, there are several possible asymmetric bifurcations with a wide range of feasibility.

We now analyze the properties of networks that were generated by taking advantage of such variability.

\section{Viscosity parameters}

Before proceeding further, we need to determine the form of the functions $\beta(H), \gamma(H)$, and $\mu_{\text {sat }}(H)$ appearing in the expression for the viscosity (Eq. (12)). We will fit them using the data provided by Eq. (6).

\footnotetext{
${ }^{4}$ In the range of radii values in which we are doing our simulations, the relative viscosity is reduced by a factor 1.5 (see Fig. 2).
} 
To determine $\beta(H)$ and $\gamma(H)$, we have proceeded as follows. First, we have repeated the procedure we followed in The design principles to obtain the branching exponents $\alpha_{i}(H)$ for different values of the hematocrit. Then, using these values for $\alpha_{i}(H)$ and Eq. (14), we obtain $\beta(H)$ and $\gamma(H)$. The results are plotted in Figs. 7a and b, respectively. The data corresponding to $\gamma(H)$ fit quite well to a straight line (Fig. 7b). For $\beta(H)$, a quadratic provides a better fit. $\mu_{\text {sat }}(H)$ was more straightforward to determine. We give a very large value to $R$ in Eq. (12), in particular $R=1 \mathrm{~cm}$, for different values of the hematocrit. The corresponding values of $\mu_{\text {rel }}$ are those we take for $\mu_{\text {sat }}(H)$, which are plotted in Fig. $7 \mathrm{c}$. We fit these data using a quadratic. A summary of our results is given in Table 1 .

\section{Blood flow simulations in optimal networks with asymmetric bifurcations}

To obtain a general idea of the effects that the introduction of hematocrit and asymmetric bifurcations produce on the blood flow through our optimal networks, we have first performed simulations in which preference is given to the occurrence of asymmetric bifurcations: when generating the optimal network, we first try one of the three asymmetric bifurcations described in Appendix A. If the bifurcation meets the corresponding feasibility criteria (see Appendix A), then the asymmetric bifurcation is implemented. Only when the asymmetric bifurcation is not possible is a symmetric bifurcation used. This is the procedure we have used to generate the results shown in Figs. 8 and 9.

Fig. 8 summarizes results for such an optimal branching network, containing asymmetric bifurcations of type NS1 and NS3 (see Appendix A for the nomenclature) and symmetric bifurcations. Fig. 9 shows the results for an optimal branching network containing asymmetric bifurcations of type NS2 and NS3 (see Appendix A for the nomenclature) and symmetric bifurcations.

Figs. $8 \mathrm{a}$ and $9 \mathrm{a}$ show how the wall shear stress varies as a function of the pressure for the respective networks. In both cases, there is what we might term a "two-branch" behavior. One of the branches exhibits the type of sigmoidal behavior observed by Pries et al. (see Fig. 3 in Pries et al., 1995 and link: Fig. 4 in Pries et al., 1998). The slope is steeper for the network containing NS1 asymmetric bifurcations. The other branch appears to have smaller values of the wall shear stress. This two-branch behavior is a consequence of the presence of asymmetric bifurcations:NS1 and NS2 yield bifurcations with daughter vessels showing very different values of the radii (Figs. 8c and 9c) and hematocrit (see Figs. 8d and 9d). In particular, one of the daughter vessels is much wider and carries much more hematocrit (see Figs. 8d and 9d) and blood flow than the other. This fact, together with the strong dependence of viscosity on both radius and hematocrit, explains why the smaller branch has a level of wall shear stress that is much smaller than the bigger branch.
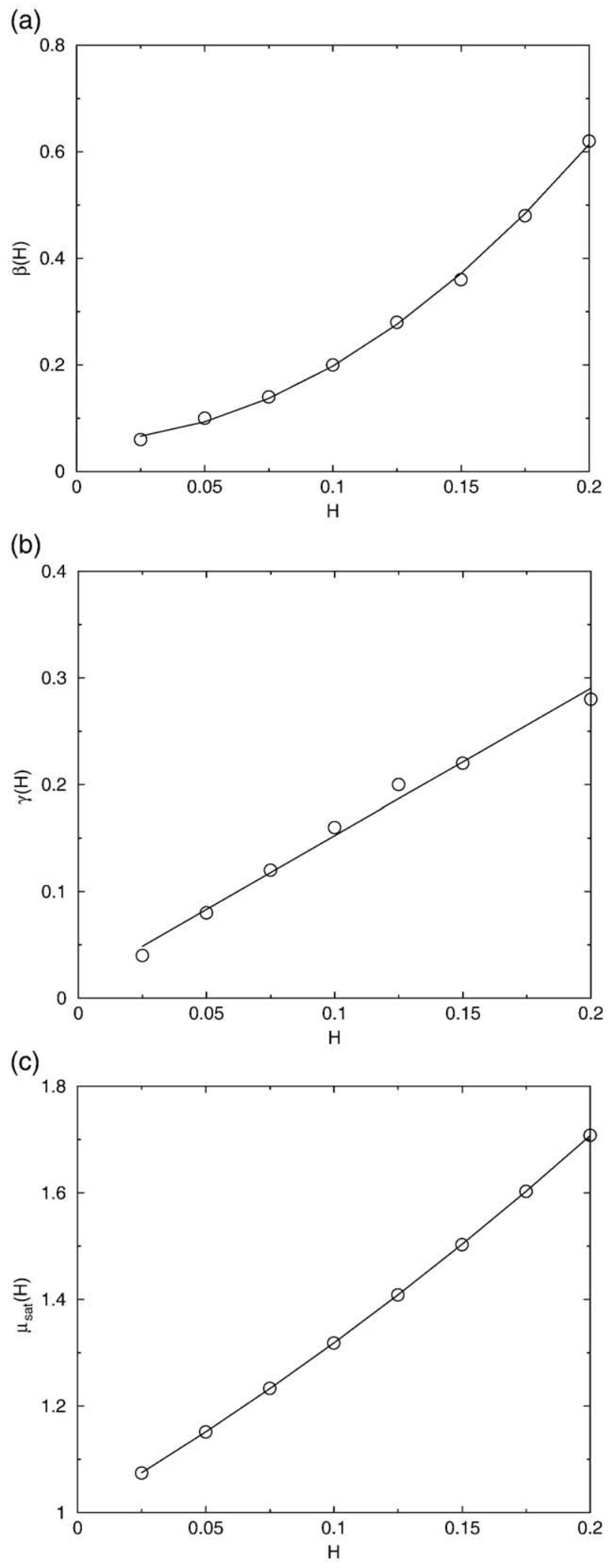

Fig. 7. These panels show the dependence of the hematocrit of (a) $\beta$, (b) $\gamma$, and (c) $\mu_{\text {sat }}$. The circles represent in each case values obtained from analysis of the experimental data (Eq. (6) see text for details). Solid lines correspond to the fits we have used: linear for $\gamma$ and quadratic for $\beta$ and $\mu_{\text {sat. The expressions }}$ we have obtained from the fitting procedure are given in Table 1. 
(a)

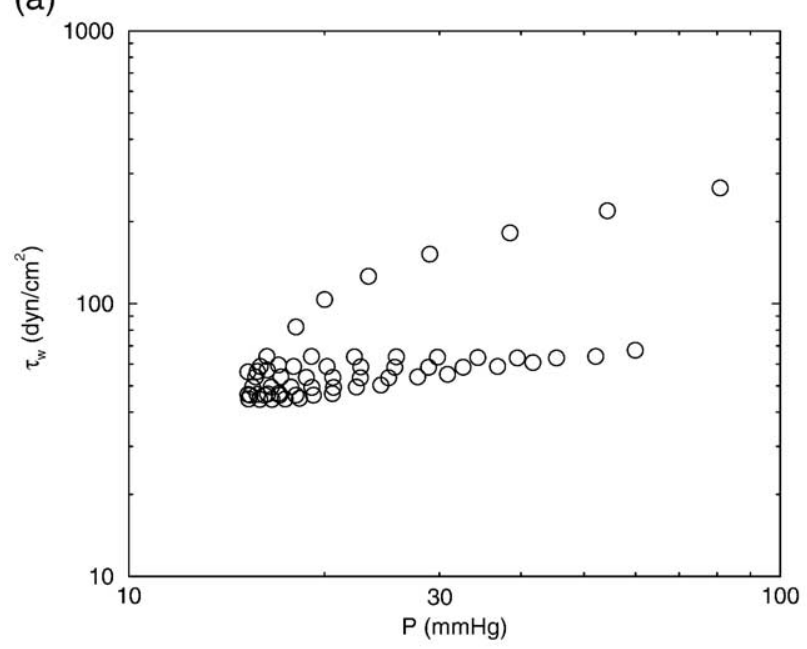

(c)

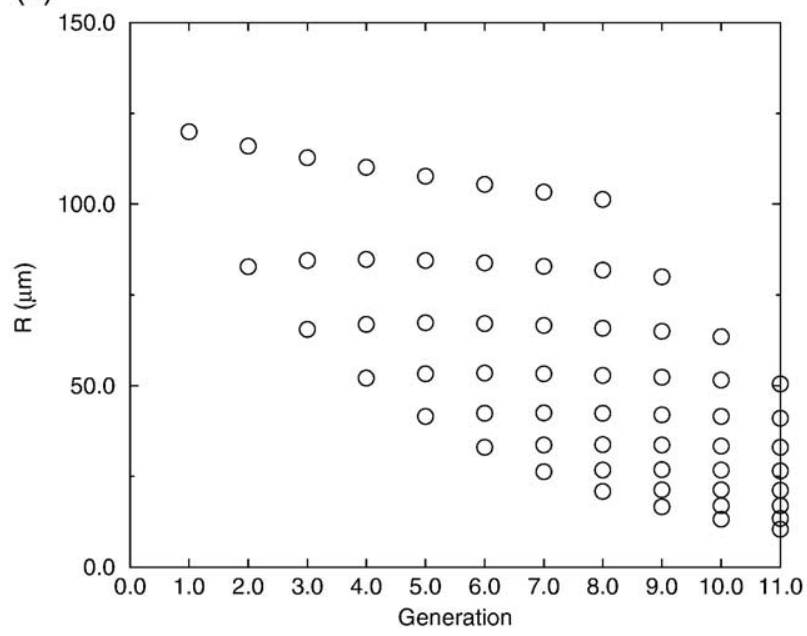

(b)

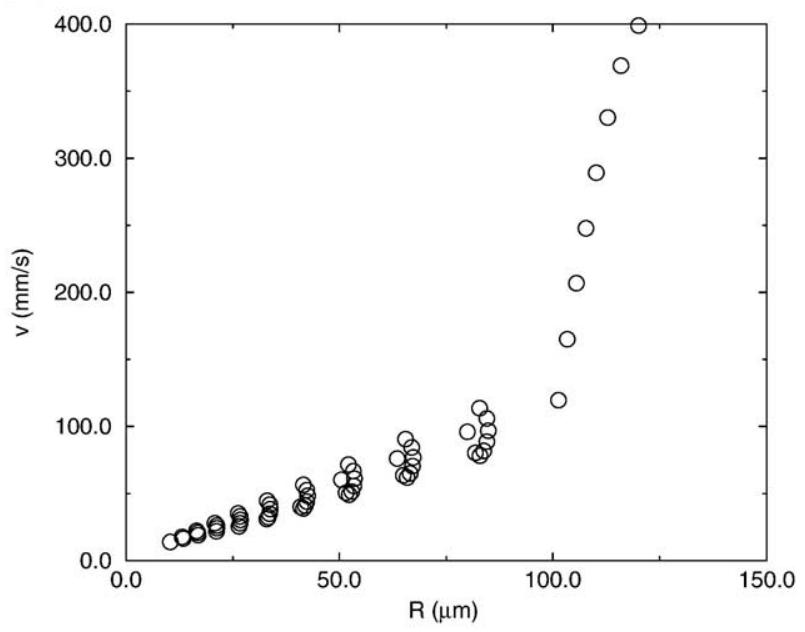

(d)

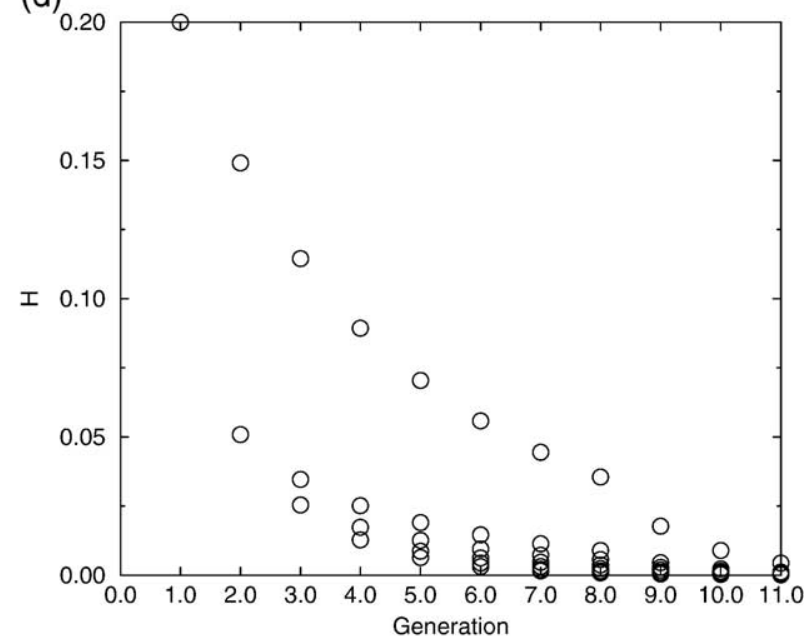

Fig. 8. Results of the blood simulations we have performed on an optimal network with three types of bifurcation: NS1-type asymmetric, NS3-type asymmetric, and symmetric (see text for details). These plots show the hemodynamic quantities corresponding to each one of the vessels, so that every circle represents an individual vessel of the vascular tree. In the case of the pressure, it is calculated in the middle point of each one of the vessels. (a) Wall shear stress as a function of pressure, (b) flow velocity as a function of radius, and (c and d) radius and hematocrit as a function of the branching generation, respectively. For these simulations: $R^{1}=120 \mu \mathrm{m}, H^{1}=0.2$, and $L^{1}=0.24 \mathrm{~cm}$. The parameter values are given in Table 1 .

Some of the smaller branches are likely to undergo vessel pruning driven by some flow-related signal, probably wall shear stress (Resnick et al., 2003). In particular, the tiny vessels generated by NS2 asymmetric bifurcations at the earlier bifurcations (see Fig. 10) have a very low level of wall shear stress which might trigger apoptosis of the endothelial cells and, consequently, vessel pruning. Since knowledge of the mechanisms involved in this process is rather poor, we have not included this phenomenon in our model. However, from the experimental results (Fig. 3 in Pries et al., 1995), we can see that levels of wall shear stress might be too low for vessel survival when $P=60 \mathrm{~mm} \mathrm{Hg}$, whereas the values of the wall shear stress corresponding to $P=20 \mathrm{~mm} \mathrm{Hg}$ seem to be feasible. Our conclusion is that any potential survival mechanism would depend not only on the wall shear stress but also on the pressure.
Now that we have a general flavor of the kind of behavior we can expect when hematocrit and asymmetric bifurcations are introduced, we present simulations performed on heterogeneous optimal networks. To build up these heterogeneous networks we proceed as follows. If the parent vessel radius $R_{\mathrm{p}}>R_{\text {sat, }}$, we choose at random between NS1 and NS2 asymmetric bifurcations. If, however, $R_{\min }<R_{\mathrm{p}}<R_{\text {sat }}$, we choose at random between a NS3 asymmetric bifurcation and a symmetric bifurcation. If the radii of the daughter vessels do not meet the feasibility criteria (see Appendix A) when an asymmetric bifurcation is attempted, we enforce a symmetric bifurcation instead.

Again, we obtain results which qualitatively reproduce the experimental observations of Pries et al. (1995). In this case, we obtain the 10 -fold reduction in the wall shear stress observed in the simulations reported by Pries et al. 
(a)

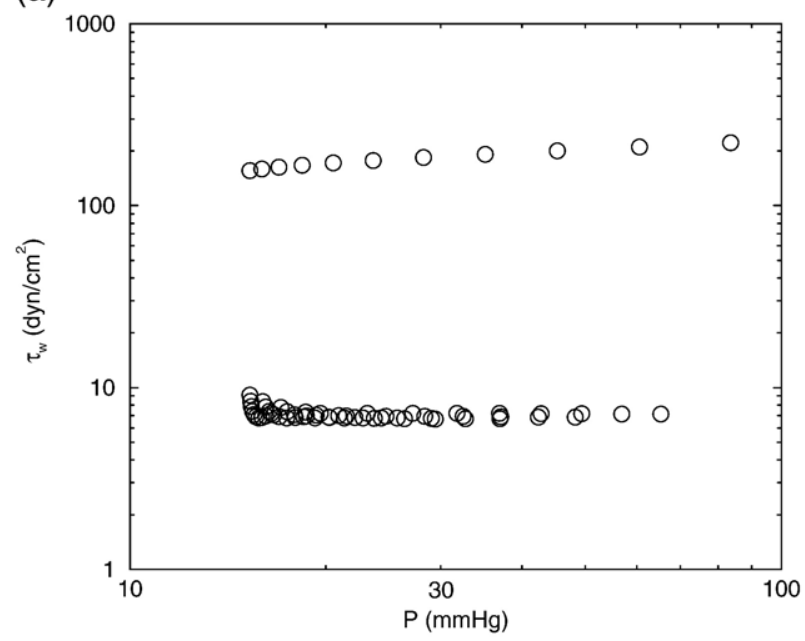

(c)

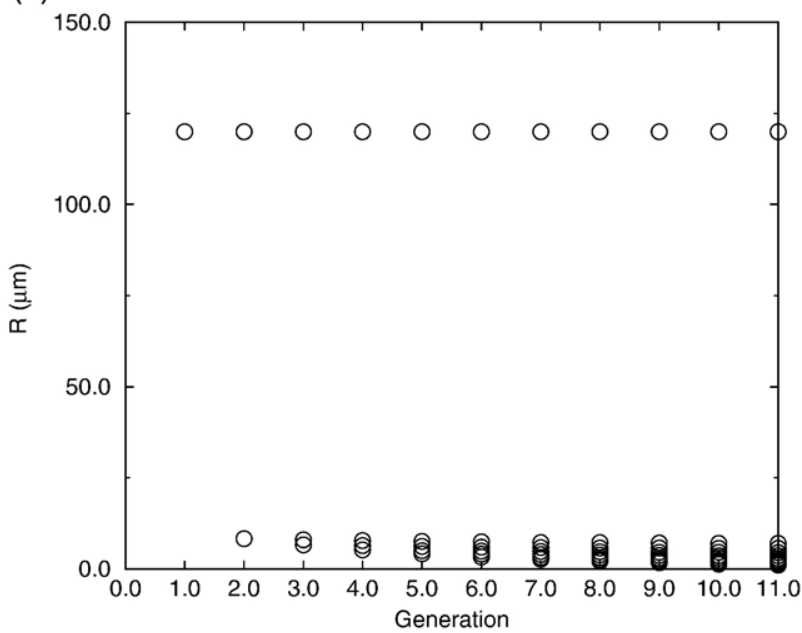

(b)

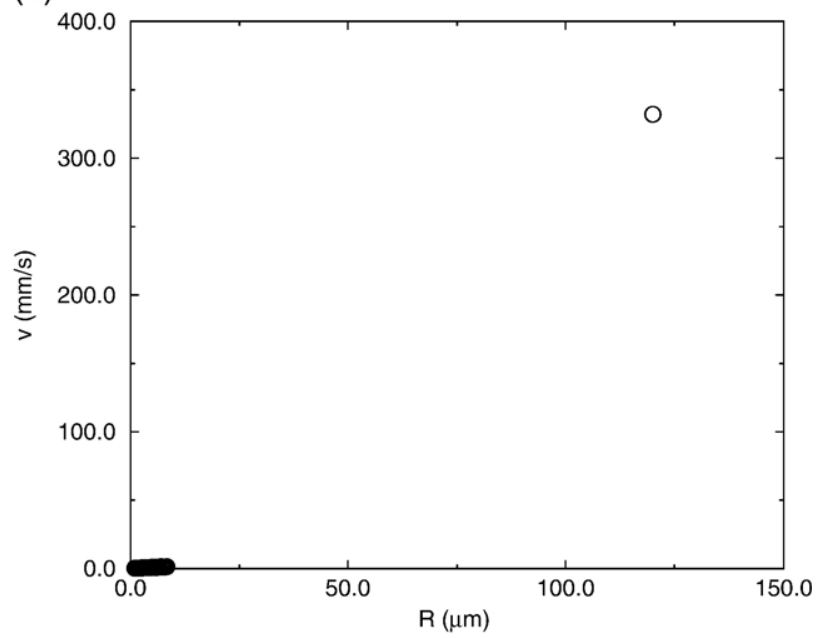

(d)

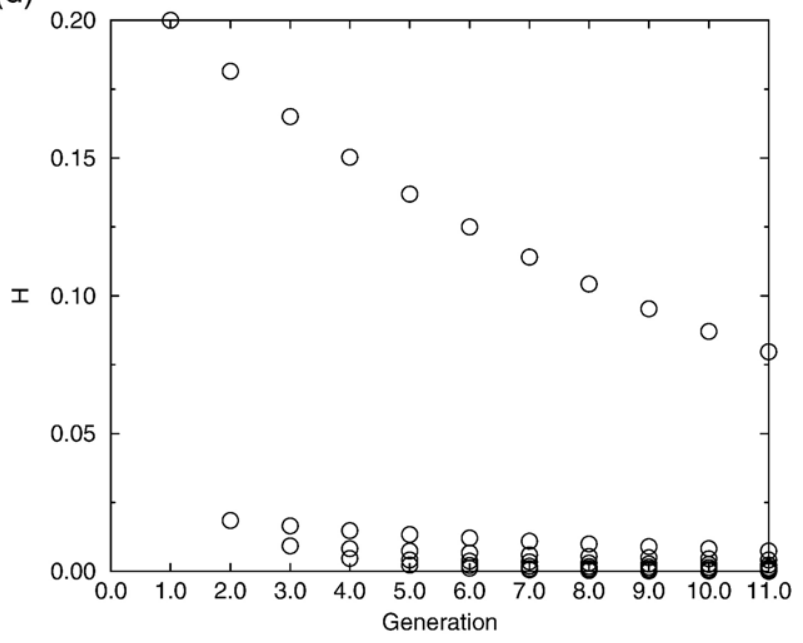

Fig. 9. Results of the simulations we have performed on an optimal network with three types of bifurcation: NS2-type asymmetric, NS3-type asymmetric, and symmetric (see text for details). These plots show the hemodynamic quantities corresponding to each one of the vessels, so that every circle represents an individual vessel of the vascular tree. In the case of the pressure, it is calculated in the middle point of each one of the vessels. (a) Wall shear stress as a function of pressure, (b) flow velocity as a function of radius, and (c and d) radius and hematocrit as a function of the branching generation, respectively. For these simulations: $R^{1}=120 \mu \mathrm{m}, H^{1}=0.2$, and $L^{1}=0.24 \mathrm{~cm}$. The parameter values are given in Table 1 .

(see Fig. 4 in Pries et al., 1998), with which we observe good quantitative agreement, except for the loss of concavity. As we have mentioned, this fact might be due to the absence in our model of a mechanism implementing wall-shear-stress-induced vascular remodeling (Resnick et al., 2003).

Regarding other hemodynamic quantities of interest, we have computed flow velocity as a function of the radius for our three different networks (see Figs. 8b, 9b, and $10 \mathrm{~b}$ ). Pries et al. (1995) reported experimental measurements of the flow velocity for radii up to $30 \mu \mathrm{m}$ (Fig. 1 in Pries et al., 1995). From Fig. 11b, we see that we obtain values for the flow velocity very similar to the observed ones in for radii between $1 \mu \mathrm{m}$ and $10 \mu \mathrm{m}$. Figs. 11a and c show that the quantitative agreement is relatively poorer for vessels with radii between 10 and $30 \mu \mathrm{m}$, specially for radii between 10 and $20 \mu \mathrm{m}$, where we obtain vessel velocities one order of magnitude bigger than the experi- ments. In the region between 20 and $30 \mu \mathrm{m}$, we obtain better quantitative agreement, as the predicted velocities are about twice as big as those observed and hence we capture the right order of magnitude. For example, for $R=$ $30 \mu \mathrm{m}, v \simeq 10 \mathrm{~mm} / \mathrm{s}$ according to the experiments (Fig. 1 in Pries et al., 1995) and $v \simeq 25 \mathrm{~mm} / \mathrm{s}$ according to our model.

Another observation about the behavior of this heterogeneous optimal network is derived from Fig. 12. Experimentally, the value of the branching exponent is obtained by collecting data for junctions over many vascular generations and averaging to obtain an estimate (Frame and Sarelius, 1995). If we were to do this, according to Fig. 12, we would obtain a figure very close to 3, i.e., Murray's law. However, Murray's law predicts a uniform value of the wall shear stress. This apparent contradiction comes from the fact that we are using a local bifurcation law (Eq. (9)), rather than a global one with an 
(a)

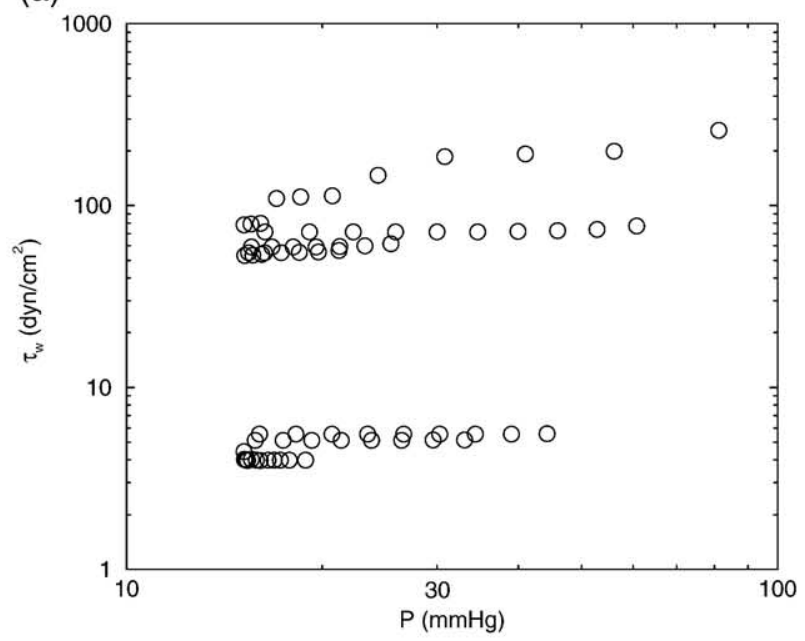

(c)

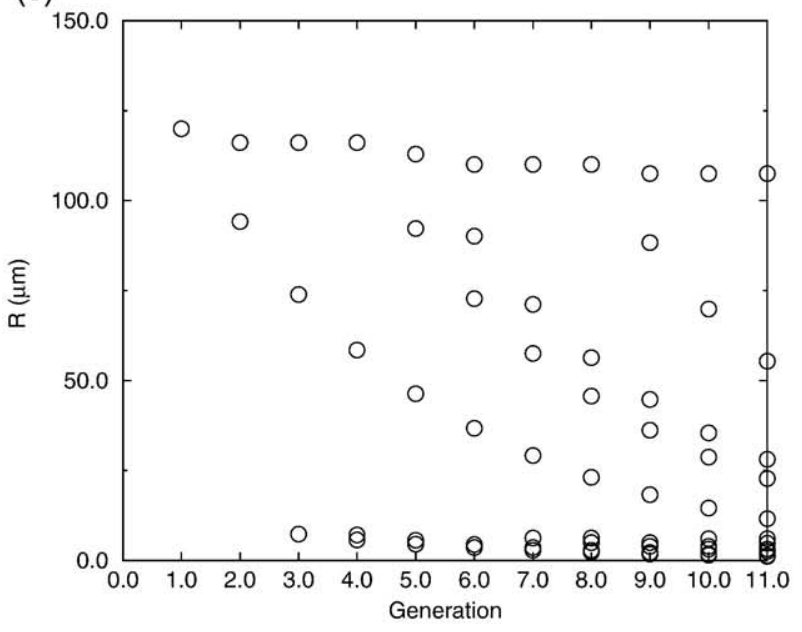

(b)

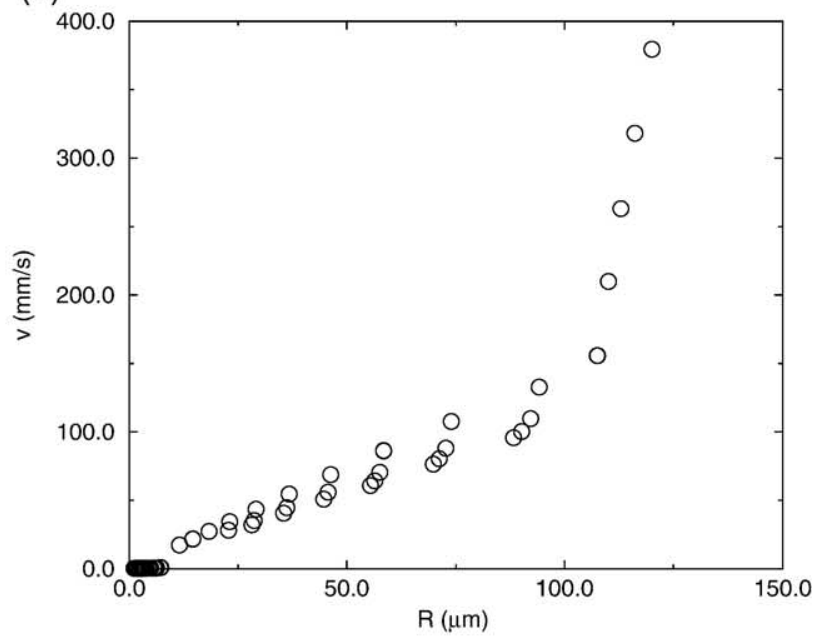

(d)

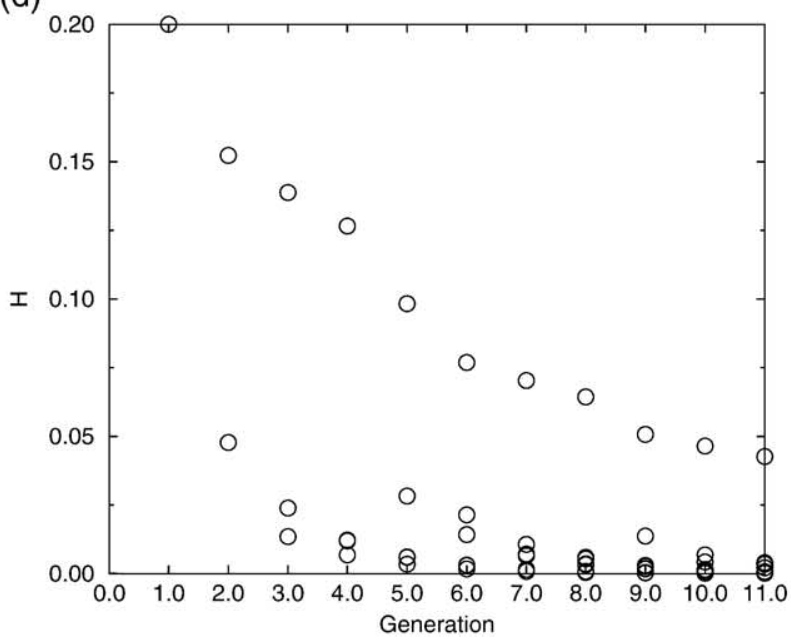

Fig. 10. Results of the simulations in an optimal heterogeneous network. To generate this network, we have chosen at random one of the possible bifurcations, either symmetric or asymmetric (see text for details). These plots show the hemodynamic quantities corresponding to each one of the vessels, so that every circle represents an individual vessel of the vascular tree. In the case of pressure, it is calculated in the middle point of each one of the vessels. (a) Wall shear stress as a function of pressure, (b) flow velocity as a function of radius, and (c and d) radius and hematocrit as a function of the branching generation, respectively. For this simulation: $R^{1}=120 \mu \mathrm{m}, H^{1}=0.2$, and $L^{1}=0.24 \mathrm{~cm}$. The parameter values are given in Table 1 .

average branching exponent. Our local bifurcation law depends on the radius and the hematocrit through the coefficients $C_{i}\left(R_{i}, H_{i}\right)$ and $\alpha_{i}\left(R_{i}, H_{i}\right)$. Karau et al. (2001) obtained similar results on studying vascular trees with heterogeneous branching exponents: even when the mean value of the branching exponent is 3 , non-uniform wall shear stress was observed. Their conclusion was that the influence on the wall shear stress in determining vessel radius is not necessarily manifested in a mean value of the branching exponent.

\section{Conclusions}

By extending the basic scheme proposed by Murray (1926) to introduce such features as complex blood rheology and hematocrit, we have formulated a design principle whose results are in good qualitative agreement with experimental observations of hydrodynamic quantities. Simulations carried out in branching networks whose geometry is determined according to our design principle reproduce experimentally observed trends of the WSS and the flow velocity (Pries et al., 1995). From the quantitative point of view, the results obtained for the wall shear stress are compatible with simulation results reported by Pries et al. (1998) and the experimental results by Pries et al. (1995). Our quantitative predictions concerning flow velocity are of the same order of magnitude as those observed in experiments (Pries et al., 1995) in several ranges of vessels radii.

We have introduced two theoretical issues in this paper which are important: heterogeneity in the network and of local bifurcation laws. By including complex blood rheology, in the form of hematocrit and radius dependent 
(a)

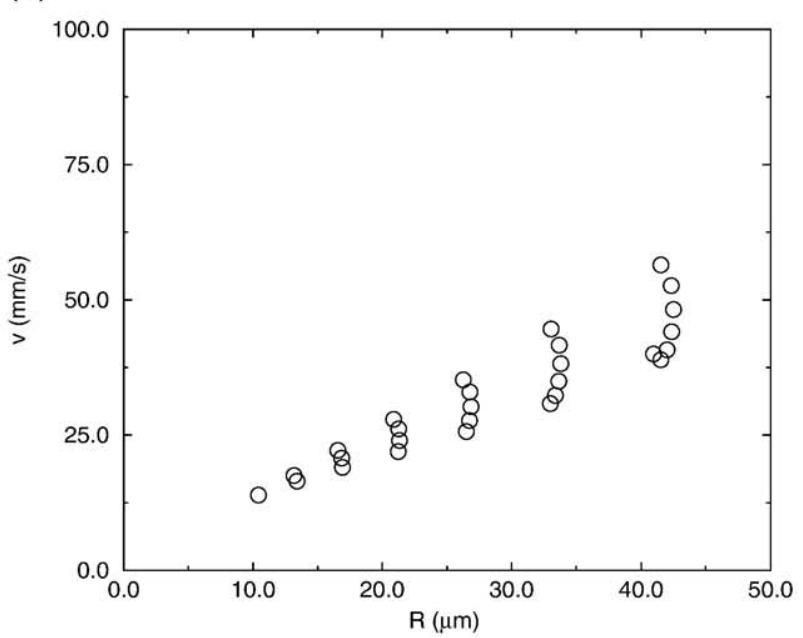

(b)

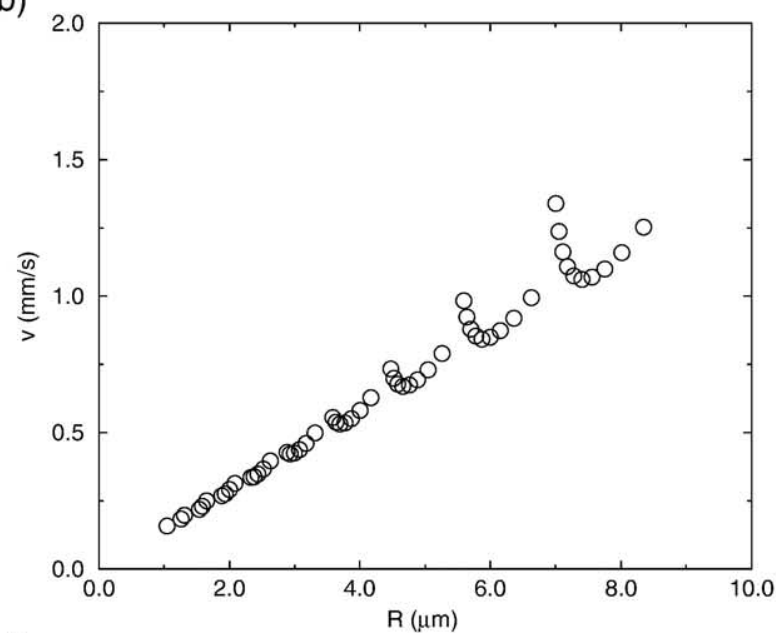

(c)

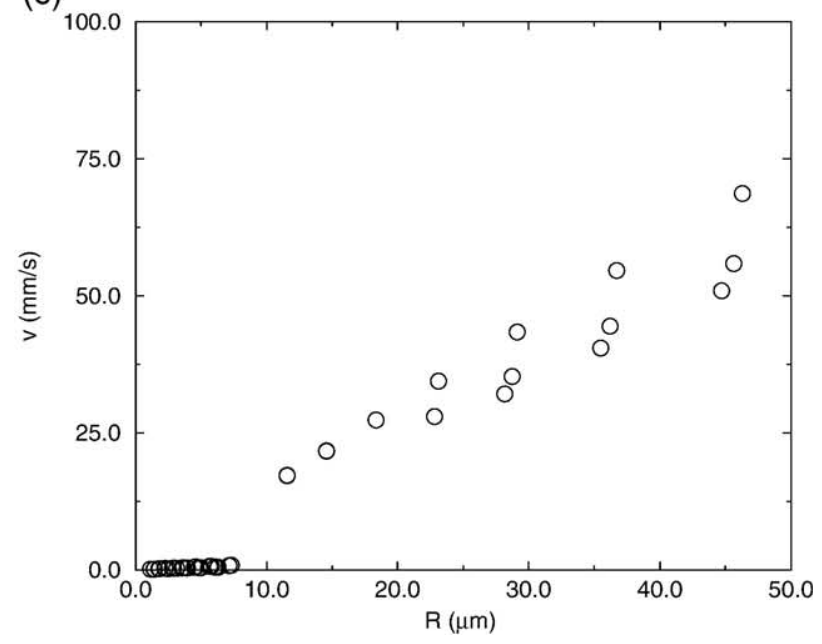

Fig. 11. Flow velocity as a function of the radius for our three different networks. These plots show the flow velocity in the range of radii in which Pries et al. (1995) carried out their experiments. (a) Optimal network containing NS1-type asymmetric, NS3-type asymmetric, and symmetric bifurcations. (b) Optimal network containing NS2-type asymmetric, NS3type asymmetric, and symmetric bifurcations. (c) Heterogeneous network.

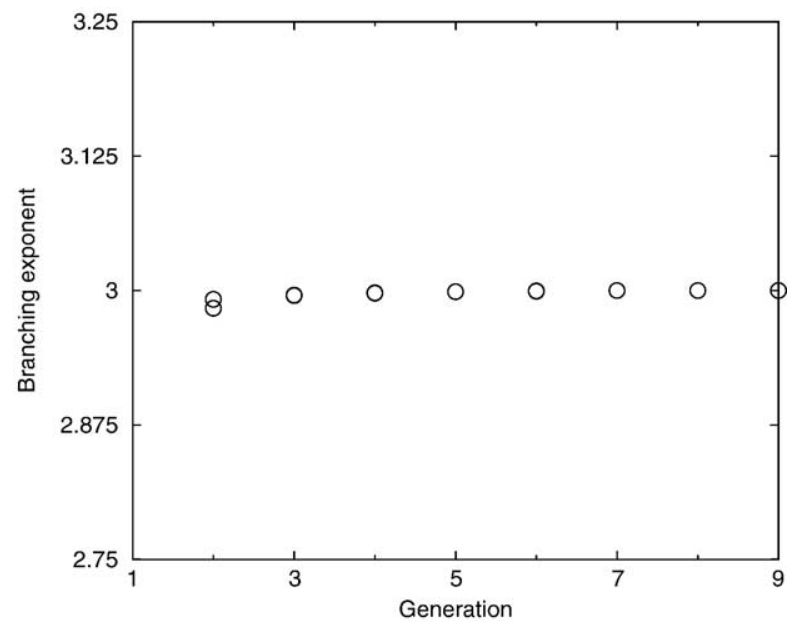

Fig. 12. Branching exponents as a function of the branching generation.

viscosity, our model naturally allows for several types of bifurcations (symmetric and various different asymmetric ones). In turn, this provides a natural way of introducing heterogeneity into our model. If the viscosity is assumed constant (Murray, 1926), our analysis in Appendix A would imply that only symmetric bifurcations are possible, leaving no room for heterogeneity.

A local bifurcation law Eq. (9) arises naturally in our model. This local bifurcation law reconciles the observation of a non-uniform wall shear stress with an average (global) value of the branching exponent very close to 3 . This result is in agreement with previous work (Karau et al., 2001). It also reconciles the experimental observation of a nonuniform wall shear stress (Pries et al., 1995) with the geometrical design principle of Gafiychuk and Lubashevsky (2001), who, from the requirement of a space filling network, deduced that the branching exponent should be equal to three.

One major weakness of our model when dealing with microcirculation is the use of Poiseuille's law. While this law accurately describes the flow in arterioles, it fails for the smaller vessels. In order to extend our methodology to describe the vasculature within tumors, we would need to account for fluid leakage from the vessel (Netti et al., 1996). We leave this as an open problem for future investigation.

\section{Acknowledgments}

The authors would like to thank Axel Pries and Timothy Secomb for helpful comments. TA thanks the Centre for Mathematical Biology, University of Oxford, where most of this work was done and the EU Research Training Network (5th Framework): "Using mathematical modelling and computer simulation to improve cancer therapy" for funding this research. MB thanks the EPSRC for funding as an Advanced Research Fellow. 


\section{Appendix A. Structure of an optimal network of rigid vessels}

In order to determine the structure of a vascular network that satisfies our optimization principle, we impose the following relationship between lumen radius and flow rate, $\dot{Q}=C R^{\alpha}$ (see Eqs. ), (10), and (11). To proceed further, let us consider the branching network depicted in Fig. 5. Application of Kirchoff's laws and Eq. (20) yields

$$
\begin{aligned}
& C\left(R_{1}, H_{1}\right) R_{1}^{\alpha\left(R_{1}, H_{1}\right)}= C\left(R_{2}, H_{2}\right) R_{2}^{\alpha\left(R_{2}, H_{2}\right)} \\
&+C\left(R_{3}, H_{3}\right) R_{3}^{\alpha\left(R_{3}, H_{3}\right)}, \\
& C\left(R_{2}, H_{2}\right) R_{2}^{\alpha\left(R_{2}, H_{2}\right)}= C\left(R_{4}, H_{4}\right) R_{4}^{\alpha\left(R_{4}, H_{4}\right)} \\
&+C\left(R_{5}, H_{5}\right) R_{5}^{\alpha\left(R_{5}, H_{5}\right)}, \\
&+C\left(R_{7}, H_{7}\right) R_{7}^{\alpha\left(R_{7}, H_{7}\right)}, \\
& C\left(R_{3}, H_{3}\right) R_{3}^{\alpha\left(R_{3}, H_{3}\right)}= C\left(R_{6}, H_{6}\right) R_{6}^{\alpha\left(R_{6}, H_{6}\right)} \\
& \frac{\mu\left(R_{1}, H_{1}\right) L_{1}}{\pi} C\left(R_{1}, H_{1}\right) R_{1}^{\alpha\left(R_{1}, H_{1}\right)-4} \frac{\mu\left(R_{2}, H_{2}\right) L_{2}}{\pi} C\left(R_{2}, H_{2}\right) R_{2}^{\alpha\left(R_{2}, H_{2}\right)-4} \\
&+\frac{\mu\left(R_{4}, H_{4}\right) L_{4}}{\pi} C\left(R_{4}, H_{4}\right) R_{4}^{\alpha\left(R_{4}, H_{4}\right)-4}=\Delta P_{\mathrm{T}},
\end{aligned}
$$

$$
\begin{aligned}
& \frac{\mu\left(R_{1}, H_{1}\right) L_{1}}{\pi} C\left(R_{1}, H_{1}\right) R_{1}^{\alpha\left(R_{1}, H_{1}\right)-4} \\
& +\frac{\mu\left(R_{2}, H_{2}\right) L_{2}}{\pi} C\left(R_{2}, H_{2}\right) R_{2}^{\alpha\left(R_{2}, H_{2}\right)-4} \\
& +\frac{\mu\left(R_{5}, H_{5}\right) L_{5}}{\pi} C\left(R_{5}, H_{5}\right) R_{5}^{\alpha\left(R_{5}, H_{5}\right)-4}=\Delta P_{\mathrm{T}},
\end{aligned}
$$

$$
\begin{aligned}
& \frac{\mu\left(R_{1}, H_{1}\right) L_{1}}{\pi} C\left(R_{1}, H_{1}\right) R_{1}^{\alpha\left(R_{1}, H_{1}\right)-4} \\
& +\frac{\mu\left(R_{3}, H_{3}\right) L_{3}}{\pi} C\left(R_{3}, H_{3}\right) R_{3}^{\alpha\left(R_{3}, H_{3}\right)-4} \\
& +\frac{\mu\left(R_{6}, H_{6}\right) L_{6}}{\pi} C\left(R_{6}, H_{6}\right) R_{6}^{\alpha\left(R_{6}, H_{6}\right)-4}=\Delta P_{\mathrm{T}},
\end{aligned}
$$

$$
\begin{aligned}
& \frac{\mu\left(R_{1}, H_{1}\right) L_{1}}{\pi} C\left(R_{1}, H_{1}\right) R_{1}^{\alpha\left(R_{1}, H_{1}\right)-4} \\
& +\frac{\mu\left(R_{3}, H_{3}\right) L_{3}}{\pi} C\left(R_{3}, H_{3}\right) R_{3}^{\alpha\left(R_{3}, H_{3}\right)-4} \\
& +\frac{\mu\left(R_{7}, H_{7}\right) L_{7}}{\pi} C\left(R_{7}, H_{7}\right) R_{7}^{\alpha\left(R_{7}, H_{7}\right)-4}=\Delta P_{\mathrm{T}},
\end{aligned}
$$

$H_{1}=H_{2}+H_{3}$,

$$
H_{2}=H_{4}+H_{5},
$$

$H_{3}=H_{6}+H_{7}$,

$\frac{H_{2}}{H_{3}}=\frac{C\left(R_{2}, H_{2}\right) R_{2}^{\alpha\left(R_{2}, H_{2}\right)-2}}{C\left(R_{3}, H_{3}\right) R_{3}^{\alpha\left(R_{3}, H_{3}\right)-2}}$,

$\frac{H_{4}}{H_{5}}=\frac{C\left(R_{4}, H_{4}\right) R_{4}^{\alpha\left(R_{4}, H_{4}\right)-2}}{C\left(R_{5}, H_{5}\right) R_{5}^{\alpha\left(R_{5}, H_{5}\right)-2}}$,

$\frac{H_{6}}{H_{7}}=\frac{C\left(R_{6}, H_{6}\right) R_{6}^{\alpha\left(R_{6}, H_{6}\right)-2}}{C\left(R_{7}, H_{7}\right) R_{7}^{\alpha\left(R_{7}, H_{7}\right)-2}}$,

where $\alpha_{i}$ is the branching exponent corresponding to $R_{i}$. We note that the outlet pressure of all vessels in the last generation is the same and that both $\alpha_{i}$ and (consequently) $C_{i}$ depend on the radius (see Eqs. (10) and (11)). Eqs. (A.4) (A.5) (A.6) (A.7) imply that

$\mu\left(R_{4}, H_{4}\right) C\left(R_{4}, H_{4}\right) R_{4}^{\alpha\left(R_{4}, H_{4}\right)-4}=\mu\left(R_{5}, H_{5}\right) C\left(R_{5}, H_{5}\right) R_{5}^{\alpha\left(R_{5}, H_{5}\right)-4} ;$
$\mu\left(R_{6}, H_{6}\right) C\left(R_{6}, H_{6}\right) R_{6}^{\alpha\left(R_{6}, H_{6}\right)-4}=\mu\left(R_{7}, H_{7}\right) C\left(R_{7}, H_{7}\right) R_{7}^{\alpha\left(R_{7}, H_{7}\right)-4}$.

For simplicity, we now assume that all the vessels in the network are equal in length $\left(L_{i}=L\right.$ constant, $i=1$, $2, \ldots, 7)$. A particular solution, and in fact the simplest one, of Eqs. (A.8) and (A.9) is $R_{4}=R_{5}=R_{6}=R_{7}$ and $H_{4}=$ $H_{5}=H_{6}=H_{7}$ since $C\left(R_{i}, H_{i}\right)$ and $\alpha\left(R_{i}, H_{i}\right)$ are identical when $R_{i}$ and $H_{i}$ are identical. In this case, Eqs. (A.4) and (A.5) admit $R_{2}=R_{3}$ and $H_{2}=H_{3}$ as a possible solution. Thus the simplest branching network that is consistent with our optimization principle is one for which all vessels in a given generation have the same radius. Referring to Eq. (9), we deduce that for such a network the radii of the subsequent generations satisfy

$$
\begin{aligned}
& R^{k+1}=\left(\frac{C^{k}}{2 C^{k+1}}\right)\left(R^{k}\right)^{\alpha^{k} / \alpha^{k+1}}, \\
& H^{k+1}=\frac{H^{k}}{2} .
\end{aligned}
$$

\section{A.1. A note concerning uniqueness}

We showed above that it is possible to construct a symmetric branching network that satisfies the restrictions imposed by Kirchoff's laws and our design principle. We now show that this network is not unique, even when we assume that the lengths of the vessels in the same generation are equal.

Consider first the case in which hematocrit is not taken into account: fix in Eqs. (A.1) (A.2) (A.3) (A.4) (A.5) (A.6) (A.7) and Eqs. (A.14) and (A.15) $H_{i}=0.45, i=1, \ldots, 7$ and neglect Eqs. (A.8) (A.9) (A.10) (A.11) (A.12) (A.13). Consider the resulting Eqs. (A.14) and (A.15), the solution to these equa- 


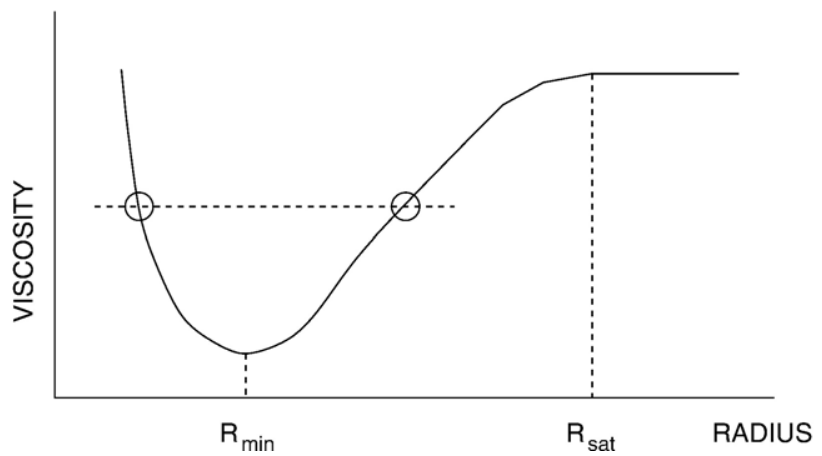

Fig. A.1. Schematic representation showing the bivalued character of the viscosity. See text for details.

tions is unique if the viscosity is a monotonic univalued function of the radius. However, as we show in Fig. A.1, there is a range of values of the radius for which the viscosity is a non-monotonous function of the radius. Therefore, bifurcations at which the daughter vessels have different radii may arise.

In order to address this question in more detail, we use the model viscosity stated in Eq. (12). Suppose that we are in the region where viscosity is a multivalued function of the radius (i.e., $R<R_{\text {sat }}$ ), so that, for instance, Eq. (A.14) may be satisfied by $R_{4}=R_{5}$ such that $R_{4}<R_{\min }<R_{5}$. The condition for this to occur is

$$
\left(\frac{R_{\min }}{R_{\mathrm{sat}}}\right)^{\gamma / 2} R_{\min }^{\beta / 2} \sqrt{\frac{1}{4+\beta}} R_{4}^{-1-\beta / 2}=R_{\min }^{\gamma / 2} \sqrt{\frac{1}{4-\gamma}} R_{5}^{-1+\gamma / 2},
$$

where we have introduced Eqs. (12) and (13) into Eq. (A.14). After some algebra we find:

$R_{4}=\left(\frac{R_{\min }^{\beta}}{R_{\mathrm{sat}}^{\gamma}} \frac{4-\gamma}{4+\beta} R_{5}^{2-\gamma}\right)^{\frac{1}{2+\beta}}$

Hence we deduce that if there are values of $R_{5} \in$ ( $\left.R_{\text {min }}, R_{\text {sat }}\right)$ for which Eq. (A.19) yields $R_{4}<R_{\text {min }}$, then it is possible to have an asymmetric bifurcation that is compatible with Kirchoff's laws and our design principle.
From Eq. (A.19), we observe that when $\gamma \leq 2$ there is a range of values of $R_{5}$ for which $R_{4}<R_{\min }$ and, hence, for which asymmetric branching may occur. However, for large values of $R_{5}$, such asymmetric branching cannot occur. Given $\gamma \leq 2$, we determine the maximum value of $\mathrm{R}_{5}$ for which asymmetric branching can occur by fixing $R_{4}=R_{\text {min }}$, to obtain

$R_{\text {crit }}=\left(\frac{4+\beta}{4-\gamma} R_{\min }^{2} R_{\mathrm{sat}}^{\gamma}\right)^{1 /(2-\gamma)}$

From Eq. (A.14) we see how changing the value of $\gamma$ affects the feasibility of the asymmetric bifurcation: for a given value of $\beta$, the asymmetric bifurcation becomes more unlikely when $\gamma$ grows, as $R_{\text {crit }}$ becomes bigger thus reducing the interval of feasibility for the asymmetric bifurcation. A similar behavior is observed when $\gamma$ is fixed and we allow variations in $\beta$ : the higher $\beta$ becomes, the more improbable the asymmetric bifurcation becomes.

The inclusion of variable hematocrit in the model yields a wider variety of possibilities. Whereas there is only one asymmetric bifurcation in the uniform hematocrit case, we have at least three different possibilities when variable hematocrit is accounted for (see Fig. A.2):

- Parent vessel with radius $R_{2}>R_{\text {sat }}$ : small daughter branch with radius $R_{4}$ such that $R_{\text {min }}<R_{4}<R_{\text {sat }}$ and large daughter vessel with radius $R_{5}>R_{\text {sat }}$. The corresponding hematocrit values for the small branch $H_{4}$ and the large branch $H_{5}$ must satisfy $H_{4}>H_{5}$ This situation is referred to as NS1 (see Fig. A.2).

- Parent vessel with radius $R_{2}>R_{\text {sat }}$ : small daughter branch with radius $R_{4}$ such that $R_{4}<R_{\text {min }}$ and large daughter vessel with radius $R_{5}>R_{\text {sat }}$. The corresponding hematocrit values for the small branch $H_{4}$ and the large branch $H_{5}$ must satisfy $H_{4}<H_{5}$. This situation is referred to as NS2 (see Fig. A.2).

- Parent vessel with radius $R_{\text {sat }}>R_{2}>R_{\min }$ : small daughter branch with radius $R_{4}$ such that $R_{4}<R_{\min }$ and
NS1

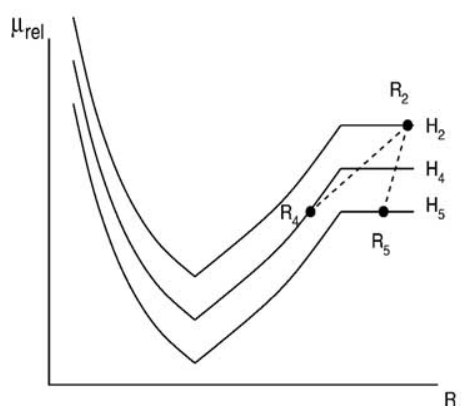

NS2

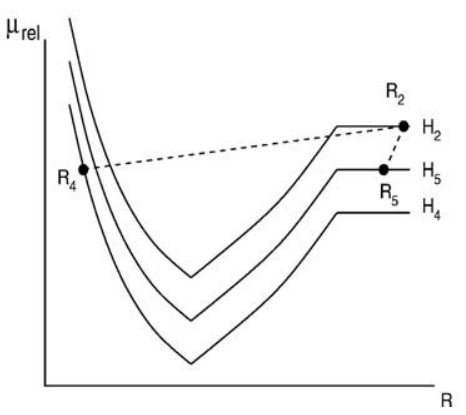

NS3

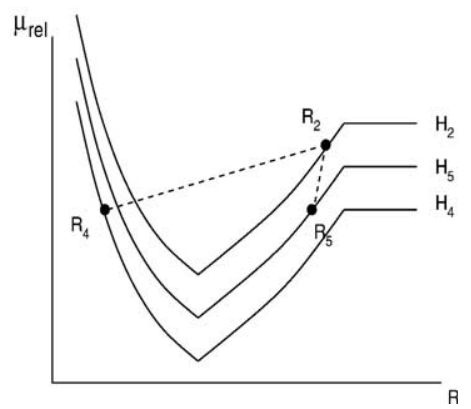

Fig. A.2. Schematic of three possible asymmetric bifurcations when hematocrit is take into account. See text for details. 
(a)

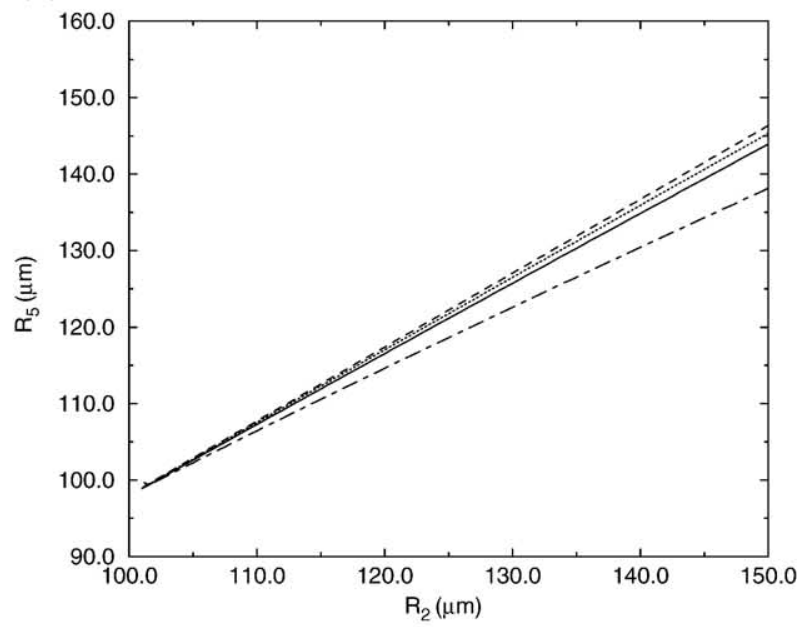

(b)

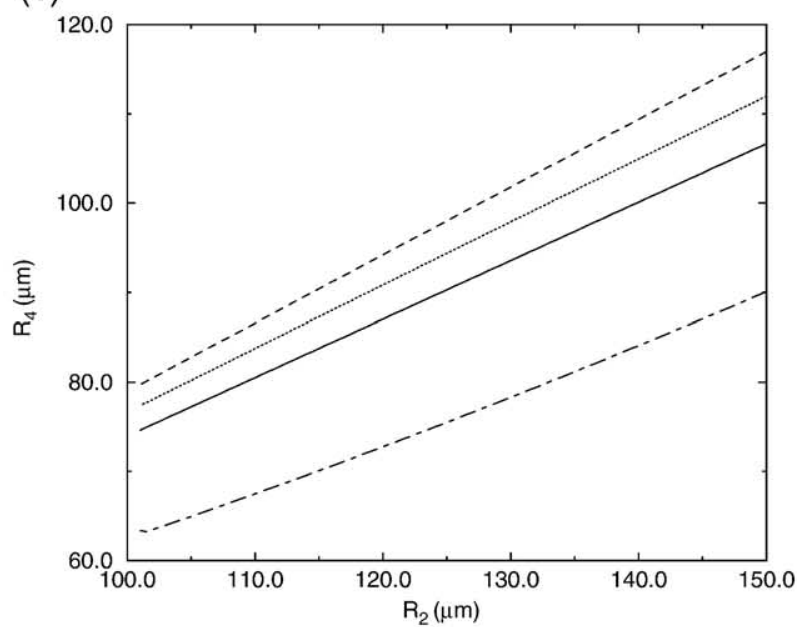

(c)

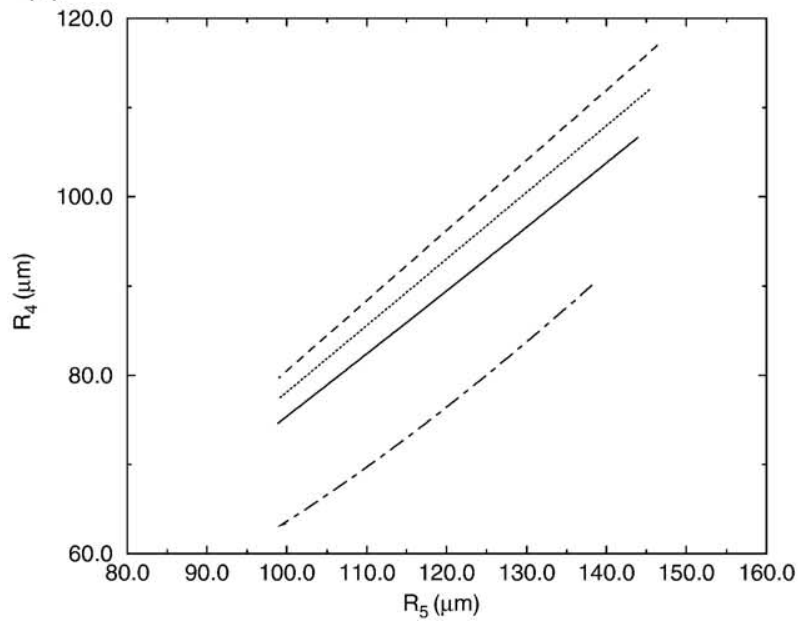

Fig. A.3. Numerical results for NS1-type asymmetric bifurcations. Dashed line corresponds to $H_{2}=0.05$, dotted line to $H_{2}=0.1$, solid line to $H_{2}=$ 0.15 , and dot-dashed line to $H_{2}=0.3$. See text for details.

large daughter vessel with radius $R_{\text {sat }}>R_{5}>R_{\min }$. The corresponding hematocrit values for the small branch $H_{4}$ and the large branch $H_{5}$ must satisfy $H_{4}<H_{5}$ This situation is referred to as NS3 (see Fig. A.2). This last case is equivalent to the (unique) asymmetric bifurcation of the uniform hematocrit case.

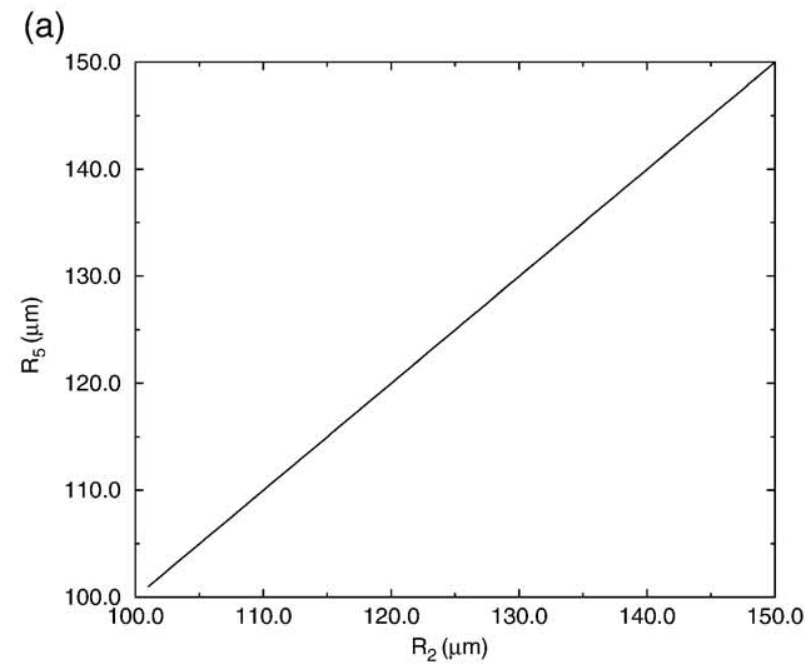

(b)

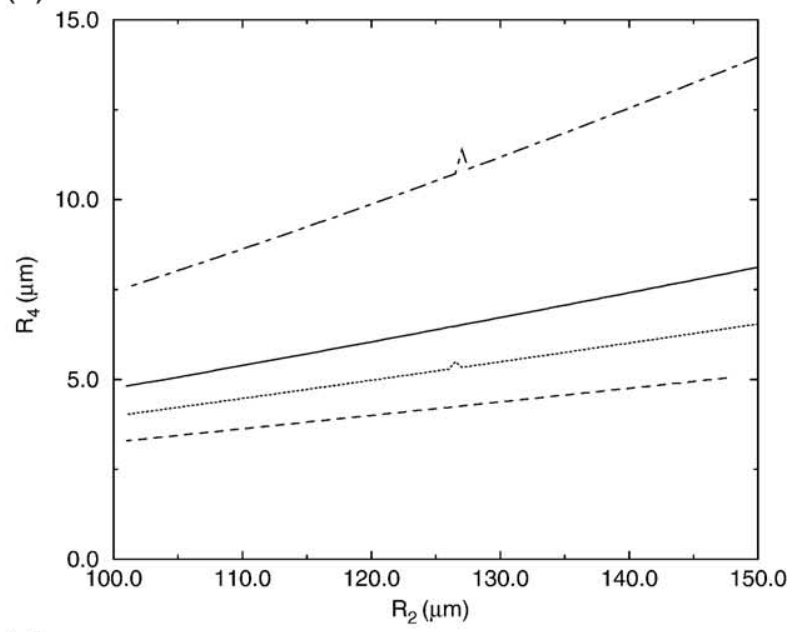

(c)

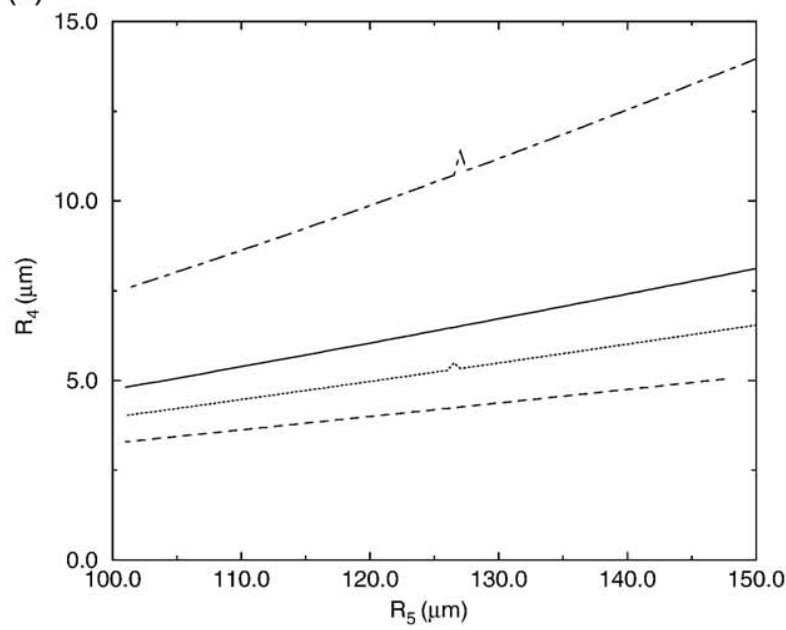

Fig. A.4. Numerical results for NS2-type asymmetric bifurcations. Dashed line corresponds to $H_{2}=0.05$, dotted line to $H_{2}=0.1$, solid line to $H_{2}=$ 0.15 , and dot-dashed line to $H_{2}=0.3$. See text for details. 
In this case, the analysis is less straightforward as for the uniform hematocrit case, but we can still make some progress. We now need to consider Eqs. (A.2), (A.12), (A.14). After some algebra, we obtain:

$\frac{H_{2}-H_{5}}{H_{5}}=\frac{R_{5}^{2}}{R_{4}^{2}\left(R_{5}, H_{5}, \dot{Q}_{2}, H_{2}\right)}\left(\frac{\pi}{2} \frac{\dot{Q}_{2} \mu_{0}^{1 / 2} \mu_{\mathrm{sat}}^{1 / 2}\left(H_{5}\right)}{\alpha_{\mathrm{b}}^{1 / 2} R_{5}^{3}}-1\right)$,

$\frac{\mu_{\mathrm{sat}}\left(H_{5}\right)}{R_{5}^{2}}=\frac{R_{2}^{\gamma\left(H_{2}-H_{5}\right)-2}}{R_{\mathrm{sat}}^{\gamma\left(H_{2}-H_{5}\right)}}\left(\frac{\mu_{\mathrm{sat}}\left(H_{2}-H_{5}\right)}{4-\gamma\left(H_{2}-H_{5}\right)}\right)$,

for NS1;

$$
\begin{aligned}
& \frac{H_{2}-H_{5}}{H_{5}}=\frac{R_{5}^{2}}{R_{4}^{2}\left(R_{5}, H_{5}, \dot{Q}_{2}, H_{2}\right)}\left(\frac{2}{\pi} \frac{\dot{Q}_{2} \mu_{0}^{1 / 2} \mu_{\mathrm{sat}}^{1 / 2}\left(H_{5}\right)}{\alpha_{\mathrm{b}}^{1 / 2} R_{5}^{3}}-1\right), \\
& \frac{\mu_{\mathrm{sat}}\left(H_{5}\right)}{R_{5}^{2}}=\frac{R_{2}^{-\beta\left(H_{2}-H_{5}\right)-2}}{R_{\min }^{-\beta\left(H_{2}-H_{5}\right)}}\left(\frac{R_{\mathrm{min}}}{R_{\mathrm{sat}}}\right)^{\gamma\left(H_{2}-H_{5}\right)} \\
& \left(\frac{\mu_{\text {sat }}\left(H_{2}-H_{5}\right)}{4+\beta\left(H_{2}-H_{5}\right)}\right) \text {, }
\end{aligned}
$$

for NS2; and

$$
\begin{aligned}
& \frac{H_{2}-H_{5}}{H_{5}}=\frac{R_{5}^{2}}{R_{4}^{2}\left(R_{5}, H_{5}, \dot{Q}_{2}, H_{2}\right)}\left(\frac{2}{\pi} \frac{\dot{Q}_{2}\left(4-\gamma\left(H_{5}\right)\right)^{1 / 2} \mu_{0}^{1 / 2} \mu_{\text {sat }}\left(H_{5}\right)}{\alpha_{b}^{1 / 2} R_{5}^{3-\gamma\left(H_{5}\right) / 2}}-1\right), \\
& \frac{\mu_{\text {sat }}\left(H_{5}\right)}{R_{5}^{2}}=\frac{R_{2}^{-\beta\left(H_{2}-H_{5}\right)-2}}{R_{\min }^{-\beta\left(H_{2}-H_{5}\right)}}\left(\frac{R_{\min }}{R_{\text {sat }}}\right)^{\gamma\left(H_{2}-H_{5}\right)}\left(\frac{\mu_{\text {sat }}\left(H_{2}-H_{5}\right)}{4+\beta\left(H_{2}-H_{5}\right)}\right),(\text { A. } 23)
\end{aligned}
$$

for NS3, respectively. In Eqs. (A.21)-(A.23), the quantity $R_{4}\left(R_{5}, H_{5}, Q\right.$ sub2, $\left.H_{2}\right)$ is given by:

$R_{4}\left(R_{5}, H_{5}, \dot{Q}_{2}, H_{2}\right)=\left(\frac{\dot{Q}_{2}-C\left(R_{5}, H_{5}\right) R_{5}^{\alpha\left(R_{5}, H_{5}\right)}}{C\left(R_{4}, H_{2}-H_{5}\right)}\right)^{1 / \alpha\left(R_{4}, H_{2}-H_{5}\right)}$.

Hence, Eqs. (A.21)-(A.24) provide a system of nonlinear equations to determine $R_{5}$ and $H_{5}$ for each case. Then, using the design principle and the corresponding conservation laws, we can obtain $R_{4}$ and $H_{4}$.

Due to the complexity of Eqs. (A.21)-(A.24), we can no longer carry out as detailed an analysis as for the uniform hematocrit case. However, we can still gain some insight from numerical solutions. We have used a NewtonRaphson method for solving these systems of equations (Press et al., 1992). This numerical study allows us to assess under which conditions we can obtain feasible bifurcations, i.e., which values of $R_{2}$ and $H_{2}$ (parent vessel radius and hematocrit) yield acceptable values of $R_{4}$ and $R_{5}$.

In the case of NS1 bifurcations, Fig. A.3a shows that acceptable values of $R_{5}$, i.e., $R_{5}>R_{\text {sat }}{ }^{5}$, for a

\footnotetext{
${ }^{5}$ Recall that $R_{\min }=0.0025 \mathrm{~cm}$ and $R_{\min }=0.01 \mathrm{~cm}$.
}

(a)

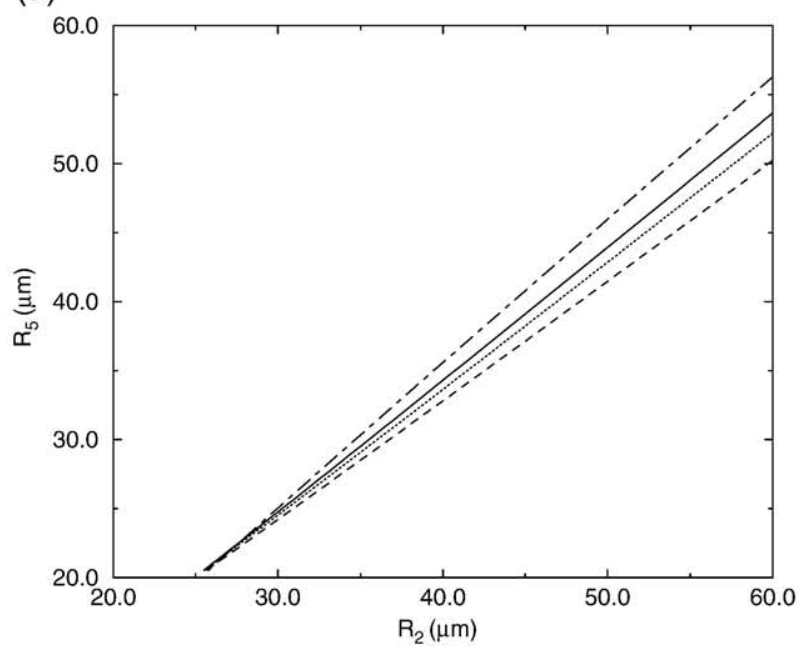

(b)

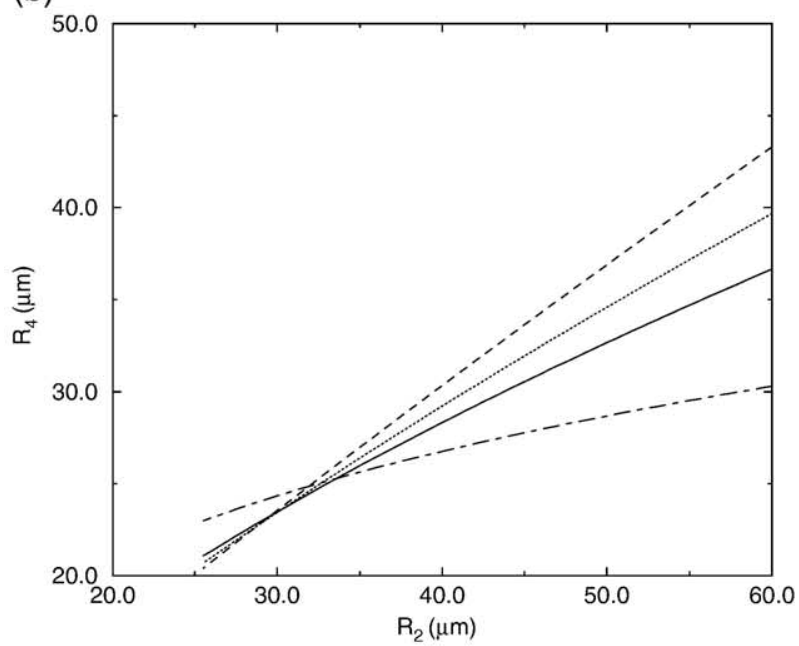

(c)

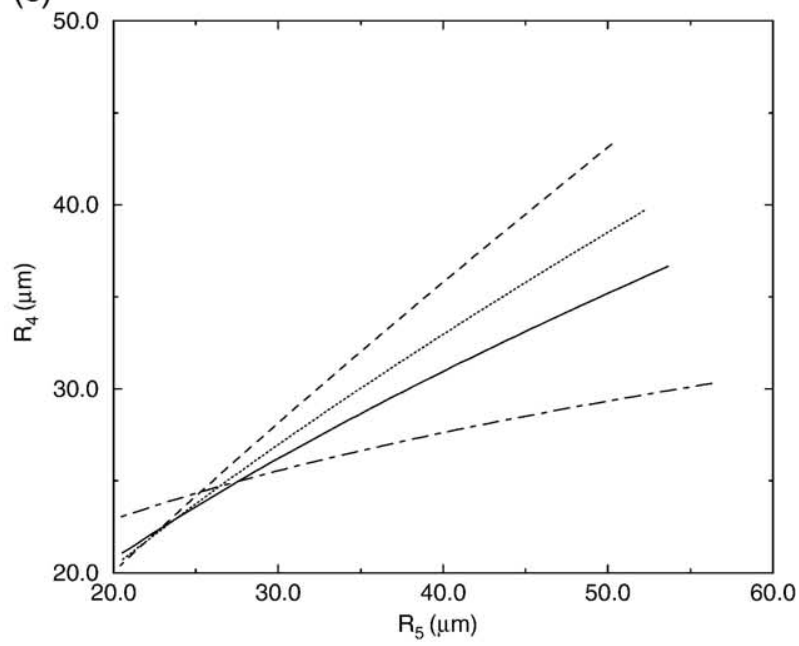

Fig. A.5. Numerical results for NS3-type asymmetric bifurcations. Dashed line corresponds to $H_{2}=0.05$, dotted line to $H_{2}=0.1$, solid line to $H_{2}=$ 0.15 , and dot-dashed line to $\mathrm{H}_{2}=0.3$. See text for details. 
wide range of values of $R_{2}$ and $\mathrm{H}_{2}$ only for values of $\mathrm{H}_{2}$ very close to $R_{\text {sat }}$ do we obtain $R_{5}<R_{\text {sat }}$. However, acceptable values of $R_{4}$, i.e., $R_{\min }<R_{4}<R_{\text {sat }}$, are obtained in a narrower range of values of $R_{2}$ and $H_{2}$. Fig. A.3b shows how, as $H_{2}$ decreases, the range of values of $R_{2}$ for which an acceptable $R_{4}$ is obtained gets smaller. Hence, the feasibility of NS1 bifurcations is determined by the smaller daughter branch. We also observe that the thinner the blood becomes (i.e., the smaller the $H$ content) the less likely this bifurcation becomes.

For NS2 bifurcations, we obtain similar results: $R_{5}$ plays no role in determining whether this type of bifurcation can actually occur (Fig. A.4a), $R_{4}$ is again the key quantity. One difference with respect to the previous case is that for NS2 bifurcations the thicker the blood becomes (i.e., the bigger the hematocrit) the more improbable this type of bifurcation becomes (Fig. A.4b)

The situation with NS3 bifurcations is again similar: a minor role is played by $R_{5}$ (Fig. A.5a) and again the bifurcation is less likely for thinner blood.

\section{References}

Frame, M.D.S., Sarelius, I.H., 1995. Microvasc. Res. 50, 301.

Fung, Y.C., 1984. Biomechanics: Circulation. Springer-Verlag, New York.

Fung, Y.C., 1990. Biomechanics: Motion, Flow, Stress and Growth. Springer-Verlag, New York.

Fung, Y.C., 1993. Biomechanics. Springer-Verlag, New York.

Gafiychuk, V.V., Lubashevsky, I.A., 2001. J. Theor. Biol. 212, 1.

Karau, K.L., Krenz, G.S., Dawson, C.A., 2001. Am. J. Phyisol. 280, H1256-H1263.

LaBarbera, M., 1990. Science 249, 992.

Murray, C.D., 1926. Proc. Nat. Acad. Sci. U. S. A. 12, 207.

Netti, P.A., Roberge, S., Boucher, Y., Baxter, L.T., Jain, R.K., 1996. Microvasc. Res. 52, 27.

Press, W.H., Teukolsky, S.A., Vetterling, W.T., Flannery, B.P., 1992. Numerical Recipes in C. Cambridge Univ. Press, Cambridge, UK.

Pries, A.R., Secomb, T.W., Gessner, T., Sperandio, M.B., Gross, J.F., Gaehtgens, P., 1994. Circ. Res. 75, 904.

Pries, A.R., Secomb, T.W., Gaehtgens, P., 1995. Circ. Res. 77, 1017.

Pries, A.R., et al., 1998. Am. J. Physiol. 275, H349-H360.

Resnick, N., et al., 2003. Prog. Biophys. Mol. Biol. 81, 177-199.

Taber, L.A., 1998. Biophys. J. 74, 109.

West, G.B., Brown, J.H., Enquist, B.J., 1997. Science 276, 122.

Zamir, M., 1976. J. Theor. Biol. 62, 227.

Zamir, M., 1977. J. Gen. Physiol. 69, 449. 